INL/EXT-16-40039 Rev. 0

September 2016

\title{
MARMOT update for oxide fuel modeling
}

Yongfeng Zhang

Daniel Schwen

Pritam Chakraborty

Chao Jiang

Larry Aagesen

Karim Ahmed

Wen Jiang

Bulent Biner

Xianming Bai

Michael Tonks

Paul Millett

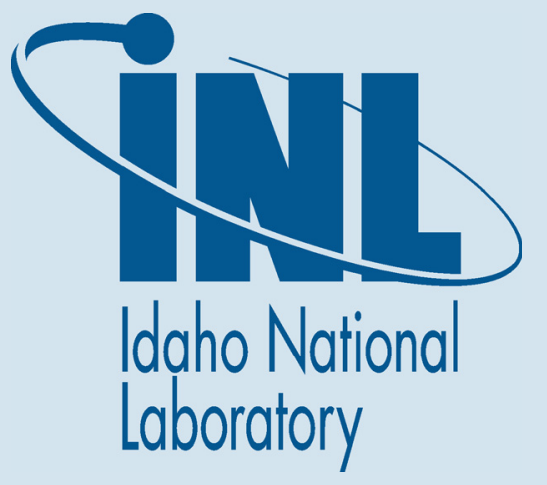




\section{NOTICE}

This information was prepared as an account of work sponsored by an agency of the U.S. Government. Neither the U.S. Government nor any agency thereof, nor any of their employees, makes any warranty, express or implied, or assumes any legal liability or responsibility for any third party's use, or the results of such use, of any information, apparatus, product, or process disclosed herein, or represents that its use by such third party would not infringe privately owned rights. The views expressed herein are not necessarily those of the U.S. Nuclear Regulatory Commission. 
INL/EXT-16-40039 Rev. 0

MARMOT update for oxide fuel modeling

\author{
Yongfeng Zhang \\ Daniel Schwen \\ Pritam Chakraborty \\ Chao Jiang \\ Larry Aagesen \\ Karim Ahmed \\ Wen Jiang \\ Bulent Biner \\ Idaho National Laboratory \\ Fuel Modeling and Simulation Department \\ Idaho Falls, Idaho 83415 \\ Xianming Bai \\ Virginia Polytechnic Institute and State University \\ Michael Tonks \\ Pennsylvania State University \\ Paul Millett \\ University of Arkansas
}

September 2016

Prepared for the

U.S. Department of Energy

Office of Nuclear Energy

Under U.S. Department of Energy-Idaho Operations Office

Contract DE-AC07-99ID13727 


\section{ABSTRACT}

This report summarizes the lower-length-scale research and development progresses in FY16 at Idaho National Laboratory in developing mechanistic materials models for oxide fuels, in parallel to the development of the MARMOT code which will be summarized in a separate report. This effort is a critical component of the microstructure based fuel performance modeling approach, supported by the Fuels Product Line of the Nuclear Energy Advanced Modeling and Simulation (NEAMS) program. The progresses can be classified into three categories: 1) development of materials models to be used in engineering scale fuel performance modeling regarding the effect of lattice defects on thermal conductivity, 2) development of modeling capabilities for mesoscale fuel behaviors including stage-3 gas release, grain growth, high burn-up structure, fracture and creep, and 3) improved understanding in material science by calculating the anisotropic grain boundary energies in $\mathrm{UO}_{2}$ and obtaining thermodynamic data for solid fission products. Many of these topics are still under active development. They are updated in the report with proper amount of details. For some topics, separate reports are generated in parallel and so stated in the text. The accomplishments have led to better understanding of fuel behaviors and enhanced capability of the MOOSE-BISON-MARMOT toolkit. 


\section{CONTENTS}

FIGURES

1 Introduction $\quad 1$

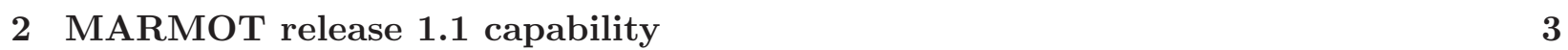

3 Stage 3 fission gas release modeling $\quad 4$

3.1 Introduction . . . . . . . . . . . . . . . . . . . . 4

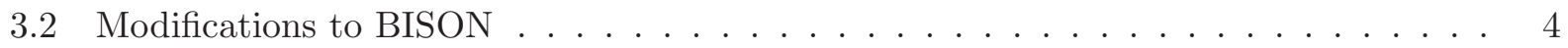

3.3 Initial Testing . . . . . . . . . . . . . . . . . . . . . . . 4

3.4 Summary and Future Plans . . . . . . . . . . . . . . . . . . . 5

4 MARMOT grain growth development $\quad 7$

4.1 Phase field model development in MARMOT considering pore dragging $\ldots \ldots$. . . . 7

4.2 Anisotropic grain boundary energies in $\mathrm{UO}_{2} \ldots \ldots \ldots \ldots$

4.2 .1 Introduction . . . . . . . . . . . . . . . . . 7

4.2 .2 Results . . . . . . . . . . . . . . . . . . . 9

4.2 .3 Implementation in MARMOT . . . . . . . . . . . . . . . 12

5 High burn-up structure formation $\quad 15$

6 Creep and fracture model development in polycrystalline solids 16

6.1 Polycrystalline creep model . . . . . . . . . . . . . . . . . . 16

6.1 .1 Example . . . . . . . . . . . . . . . . . . . 18

6.2 Comparison between Phase-field and XFEM models for brittle fracture . . . . . . . 20

7 Thermodynamics of solid fission products in $\mathrm{UO}_{2} \quad 24$

7.1 Introduction . . . . . . . . . . . . . . . . . . . . 24

7.2 Review of binary phase diagrams . . . . . . . . . . . . . . . 24

7.3 Density Functional Theory calculations of thermodynamic properties . . . . . . . 25

7.4 Results . . . . . . . . . . . . . . . . . . . 26

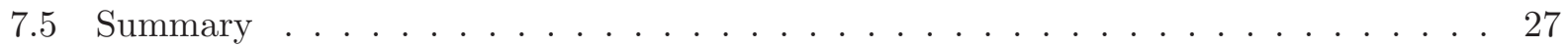

8 Microstructure-based thermal conductivity 29

8.1 Introduction . . . . . . . . . . . . . . . . . . . . . 29

8.2 Work during FY $2016 \ldots \ldots \ldots \ldots \ldots \ldots \ldots$

8.2.1 Effect of point defects on thermal conductivity . . . . . . . . . . . . . 29

8.2.2 Validation on the effect of grain boundaries . . . . . . . . . . . . . . . . . . . . . . . .

8.3 Summary . . . . . . . . . . . . . . . . . . . . . . 32

8.4 Future work . . . . . . . . . . . . . . . . . . . 32

9 Center hole formation in fast reactor fuels 33

9.1 Introduction . . . . . . . . . . . . . . . . . . 33

9.2 Simulation methodology . . . . . . . . . . . . . . . . . 34

9.3 Results and Discussion . . . . . . . . . . . . . . . . . . 36

9.4 Conclusions . . . . . . . . . . . . . . . . . . . . . 39 
11 Summary

12 References 


\section{FIGURES}

1 Schematic showing the NEAMS FPL plan to develop materials models for fuel performance codes based on microstructure rather than burn-up. Note that the variables and models listed in the figure are not a complete list. . . . . . . . . . . . . . . .

2 Schematic illustrating the multiscale approach being taken by the NEAMS FPL to develop materials models for fuel performance codes based on microstructure rather than burn-up.

$32 \mathrm{D}$ axisymmetric simulations of a single $\mathrm{UO}_{2}$ fuel pellet. Fuel pellet centerline is at left, radial exterior surface at right in each subfigure. In Case 1, fission gas can only escape through the radial exterior surface, so no percolated pathway exists, and $F_{c}>1$, which is unphysical. In Case 2, fission gas can also escape through the top and bottom, so percolation happens early in the simulation, and $F_{c}=0.5$ is maintained throughout. . . . . . . . . . . . . . . . . . . . . . . and the $\gamma$-surface for the $<110>\Sigma 3$ STGB (right) . . . . . . . . . . . . 9

$5 \quad$ GB energy $\left(\mathrm{J} / \mathrm{m}^{2}\right)$ as a function of rotation angle for $(\mathrm{a})<100>$, (b) $<110>$ and (c) $<111>$ twist GBs, and (d) $<100>$, (e) $<110>$ and (f) $<111>$ STGBs. The dots are obtained by MD simulations, and the curves are fitted using RSW functions. Note that for each 1D subset, the RSW function may be used different times with different numbers of fitting parameters (shown as insets in the figures) . . . . . . . . .

6 GB energy $\left(\mathrm{J} / \mathrm{m}^{2}\right)$ surfaces for $2 \mathrm{D}(\mathrm{a})<100>$, (b) $<110>$ and $(\mathrm{c})<111>$ tilt subsets as functions of angles $\xi$ and $\eta$, in units of degrees. The pink dots are MD results (in addition to those used in 1D subsets) used for fitting; some of them are hidden below the surface. . . . . . . . . . . . . . . . . . . 10

7 Statistics of fitting errors in term of percent deviation from MD value. . . . . . . . 11

8 GB energy $\left(\mathrm{J} / \mathrm{m}^{2}\right)$ as a function of rotation angle for the $<210>$ twist subset. The dots are from MD simulations; the curve is predicted by the $5 \mathrm{D}$ model. On the right the prediction error in reference to the MD results is plotted. . . . . . . . . . . . . 12

9 Parameters used in the $5 \mathrm{D}$ model for $\mathrm{UO}_{2} \ldots \ldots \ldots \ldots \ldots$

10 Relative GB energy $\left(\mathrm{J} / \mathrm{m}^{2}\right)$ fpr a polycrystalline sample calculated in MARMOT using the $5 \mathrm{D}$ model . . . . . . . . . . . . . . . . . . . . 14

11 (a) Geometry of the square domain; (b) Grain boundary order parameter. . . . . . . 19

12 (a) Chemical potential and (b) concentration distribution at $t=100$ sec. $\ldots \ldots$. . 19

13 Vacancy flux along (a) $\mathrm{x}$ and (b) y directions . . . . . . . . . . . . . . 19

14 (a) Spatial distribution of equivalent creep strain; (b) Temporal evolution of volume averaged equivalent creep strain. . . . . . . . . . . . . . . . 20

15 Symmetric center crack: (a) Geometry; (b) Comparison of stress-strain. . . . . . . . 20

16 (a) Geometry of the inclined crack. Deviation from self similarity (b) phase-field fracture and (c) X-FEM. In (c), METHOD 1 and 2 are based on configurational force and maximum circumferential stress from interaction integral. . . . . . . . . . 21

17 Schematic of the Bittencourt problem . . . . . . . . . . . . . . . 22

18 (a) Experiment, (b) Phase-field fracture and (c) X-FEM . . . . . . . . . . . . . 22

19 (a) Experiment, (b) Phase-field fracture and (c) X-FEM . . . . . . . . . . . . 22

20 Literature review of the phase diagrams of binary systems between the major solid fission products of $\mathrm{U}-235 . \ldots \ldots \ldots \ldots \ldots \ldots$

21 Example phase diagrams of types A to D. . . . . . . . . . . 26 
22 Two versions of the Mo-Tc phase diagram. Version 1 is from REF.Massalski1990 and version 2 is from REF.Haines1980 . . . . . . . . . . . . . 27

$23 \Delta H_{\text {chem }}$ vs. $\Delta H_{\text {strain }}$ plot for the binary sub-systems of Zr-Mo-Nd-Ru-Cs-Ce-SrBa-La-Tc-Pd-Pr-Y. . . . . . . . . . . . . . . . . . . . 28

$24 \Delta H_{\text {chem }}$ vs. $\Delta H_{\text {strain }}$ plot for the binary sub-systems of Mo-Ru-Tc-Pd. . . . . . . . 28

25 (a) Schematic of a $2 \mathrm{D}$ simulation cell for polycrystalline $\mathrm{UO}_{2}$. The grain boundaries are decorated by refined meshes. A temperature gradient is set up from left (hot) to right (cold). (b) Thermal conductivities of $\mathrm{UO}_{2}$ samples with different grain sizes as function of temperature. The dash lines are MARMOT results without corrections for stoichiometry and porosity. The solids lines are after corrections. . . . . . . . .

26 Microscopy images of both lenticular pores and prolate (bullet) shaped pores during thermal-gradient-driven migration in nuclear fuel. Generally, observations show that lenticular pores develop in the inner regions of the pellet and prolate pores develop at the circumference of the columnar grain region, in the middle or outer sections of

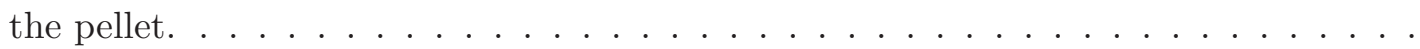

27 With an assumed temperature profile in the radial direction (top), the transport velocity of a pore due to the evaporation-condensation mechanism in the radial direction, as derived by Sens in REF.35, is plotted (bottom). Note that in these plots, the positive radial direction is along the $\mathrm{x}$-axis, and a negative transport velocity results in a pore migration toward the fuel centerline. . . . . . . . . . .

28 The simulations in this work used linear equations to describe the transport velocity in the radial direction. These linear equations overlap with the Sens' model in REF.35 at different radial locations, with the top plot showing Eq. 26 and the bottom plot showing Eq. 27. The grey shaded boxes correspond with the simulation domain size in the radial direction $(=256 \mu \mathrm{m}$, with a $512 \times 128$ grid $) \ldots \ldots . . . .38$

29 Simulation snapshots of pore migration using Eq. 26 as the transport velocity field. The top-to-bottom images correspond to 0, 1000, 2000, and 3000 simulation steps, respectively. The left side of the domain corresponds to the fuel centerline when using Eq. 26. These images were taken from a simulation that used the larger grid

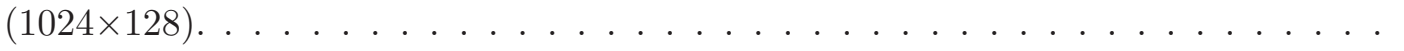

30 The velocity of a migrating pore is plotted versus the pore's radial position. The blue data points correspond to velocity values measured from a simulation, as explained in the tex, using Eq.26 for the transport velocity and a grid size of $512 \times 128$. Regardless of the fact that the pore has changed shape to a lenticular morphology, the pore velocity matches very closely to the prescribed model. . . . . . . . . . . .

31 Simulation snapshots of pore migration using Eq. 27 as the transport velocity field. The top-to-bottom images correspond to 0, 1000, 2000, and 3000 simulation steps, respectively. These images were taken from a simulation that used the larger grid $(1024 \times 128) \ldots \ldots \ldots \ldots \ldots \ldots \ldots \ldots$

32 The velocity of a migrating pore versus the pore's radial position for a pore that transforms into a prolate (e.g., bullet) shaped morphology as shown in Fig. 30 when Eq. 27 is used as the transport velocity. . . . . . . . . . . . . . . .

33 The $\mathrm{x}$ - and $\mathrm{y}$-dimensions of a migrating pore that transforms into (top) a prolate morphology and (bottom) a lenticular morphology. These values were measured from simulations using Eq. 27 and Eq. 26 for the top and bottom plots, respectively. The blue dots correspond to the pore's y-dimension and the red dots correspond to the pore's x-dimension. . . . . . . . . . . . . . . . . . . . 


\section{Introduction}

Nuclear fuels and claddings operate in reactor environments combining high temperature/temperature gradient and neutron irradiation with relatively long service times. During operation, the chemistry of fuels (claddings) changes due to fissile events and/or corrosion, and their microstructures evolve due to the evolution of radiation produced defects, segregation and precipitation of fission products, and mechanical deformation. These changes in fuel chemistry and microstructure also change fuel properties, directly impacting fuel performance and safety. For different types of fuels or same type fuels with different initial microstructures, they may experience distinct evolution in chemistry and microstructure, so that the experience obtained with one fuel may not be directly borrowed for another. In traditional fuel performance modeling, empirical materials models are usually used. Most of times these models are fitted to experimental data and correlated with temperature and burn-up. However, burn-ups by itself is insufficient to represent the evolution in chemistry and microstructure. Other factors such as fission rate, fuel type, and initial microstructure can play important role as well. Therefore, the using of empirical materials models can lead to inaccurate predictions of transient fuel properties and thus unreliable fuel performance modeling results for new types of fuels, or fuels with different microstructures. To address this, a microstructure based fuel performance modeling approach is taken by the Fuels Product Line of the Nuclear Energy Advanced Modeling and Simulation (NEAMS) program, targeting a microstructure-based fuel performance modeling approach in place of the burn-up approach.

In the new approach taken by NEAMS, the microstructure of fuels are represented by a list of state variables which evolve with time according to operation conditions including temperature and fission rate. Several examples of the state variables are grain sizes (average and distribution), densities of lattice defects, porosity, and grain boundary bubble coverage. For each state variable, a mechanistic model will be developed to predict its evolution. The transient fuel properties will be estimated, by using another set of mechanistic models, based on the current microstructure, i.e., the state variables. This approach is illustrated in Fig. 1.

The development of mechanistic models for microstructure evolution and microstructure-property correlation needs large amount of data as inputs, preferably from experiments. Unfortunately, much of the needed data is yet to be obtained and could be difficult and expensive experimentally. In addition to the missing data, many of the fundamental mechanisms that govern the evolution of certain microstructure features and the microstructure-property correlation are yet to be explored. To mitigate this difficulty, under NEAMS a multiscale modeling and simulation approach is adopted. In this approach, first principles calculations are used to investigate basic mechanisms that take place within the bulk of the material, and to quantify material properties defined by these mechanisms. Molecular dynamics simulations are used to investigate phenomena involving lattice defects such as interfaces in the materials and the corresponding properties, as well as dynamic properties such as mobilities. Other atomistic methods such as kinetic Monte Carlo are also used as needed. The information regarding the critical mechanisms and material property values is then used to develop mesoscale models that predict the co-evolution of the microstructure and the effective macroscale material properties. These data are used to inform the development of the various mechanistic models required to complete the microstructure-based set of materials models. The multiscale modeling and simulation approach is summarized in Fig. 2.

A critical component of this multiscale modeling and simulation approach is to develop the phase field MARMOT code which is used to represent the microstructure and its evolution, and to predict the current fuel properties [1]. The MARMOT code is based on the open source Multiphysics Object-Oriented Simulation Environment (MOOSE) [2]. It takes inputs from atomistic scale, describes mesoscale fuel behaviors, and is used to develop mechanistic materials models that 


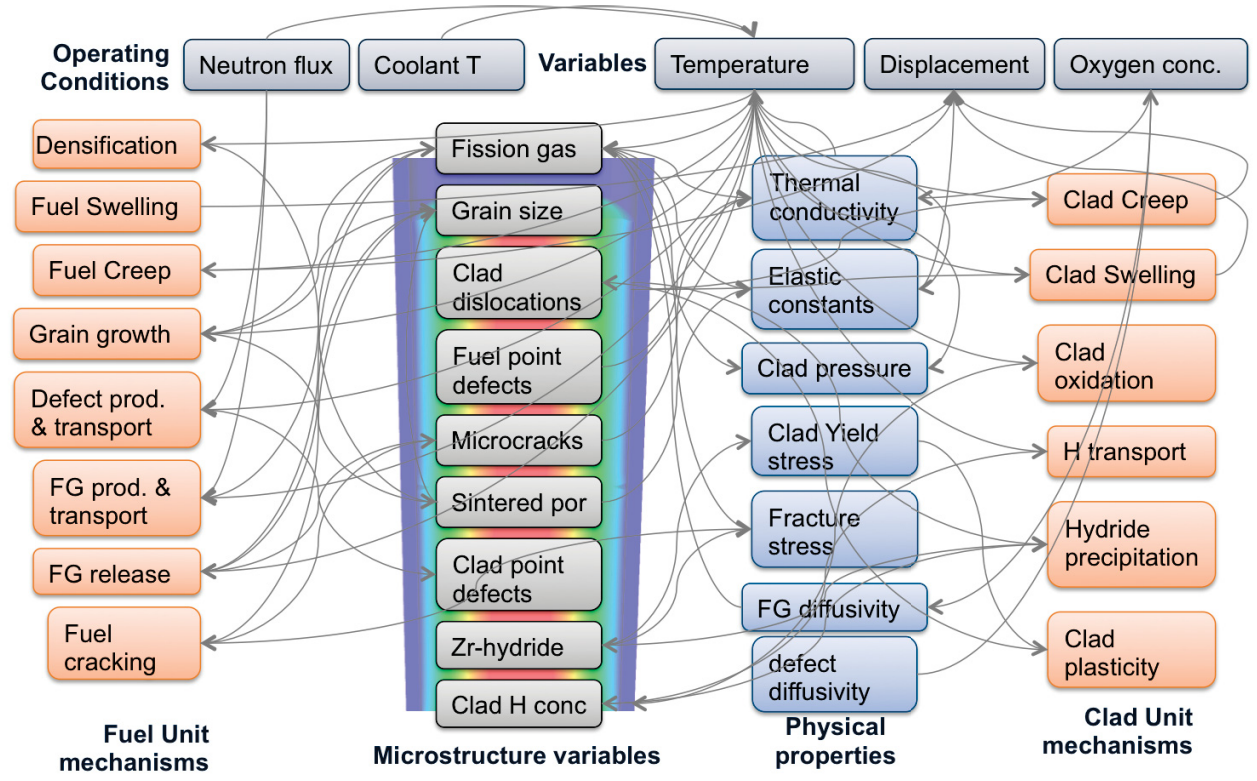

Figure 1: Schematic showing the NEAMS FPL plan to develop materials models for fuel performance codes based on microstructure rather than burn-up. Note that the variables and models listed in the figure are not a complete list.
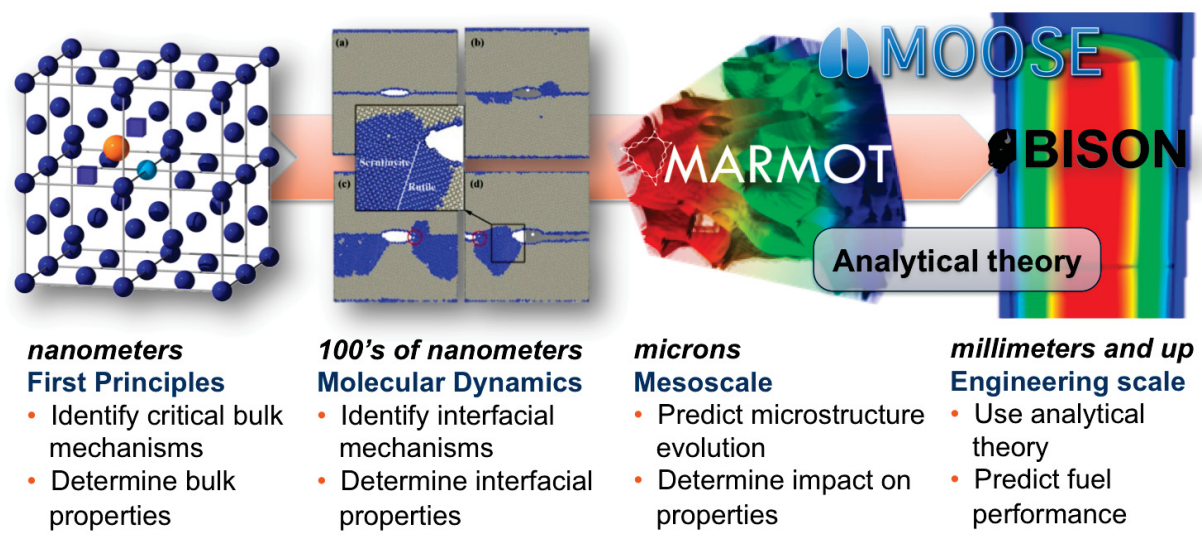

Figure 2: Schematic illustrating the multiscale approach being taken by the NEAMS FPL to develop materials models for fuel performance codes based on microstructure rather than burn-up.

can be used in the engineering scale fuel performance code BISON [3]. The primary task of NEAMS FPL is to develop the MOOSE-BISON-MARMOT (MBM) toolkit using the approach shown in Fig.2.

In the previous years, several summary and assessment reports have been generated regarding the capabilities and materials models that are being developed in MARMOT $[4,5]$. Here, the progresses made in FY16 are summarized with proper amount of details. For certain topics, a separated report with rich details is being created and will be cited here. In those cases, brief introductions will be given for completeness. 


\section{MARMOT release 1.1 capability}

At the end of fiscal year 2016 release 1.1 of the MARMOT mesoscale simulation code was published. A brief summary of the code's capabilities will be given here. For a comprehensive overview of the capabilities implemented in MARMOT please consult report INL/EXT-16-39975 Assessment of MARMOT Release 1.1: A Mesoscale Fuel Performance Code [5].

MARMOT builds on the capabilities from the phase_field and tensor mechanics modules, along with the basic heat conduction capability from the heat_conduction module, to model the coevolution of microstructure and properties in fuel and cladding materials. While the modules are open source and openly accessible using Github, MARMOT access is not open. There is no license or cost associated with MARMOT access; users simply have to contact a member of the MARMOT development team and request access.

The phase_field module in MOOSE contains the necessary tools to solve the partial differential equations for the phase field method that define the microstructure variable evolution to minimize the overall free energy. The evolution of non-conserved order parameters (representing phase regions and grains) is governed by the Allen-Cahn equation and conserved order parameters (representing concentrations) are evolved using the Cahn-Hilliard equation.

Significant contributions developed for MARMOT include a modular free energy system with automatic symbolic differentiation support. Through this automatic differentiation system we achieve a significant reduction in developer time and remove a source of developer errors that are difficult to track down and debug. The resulting models offer optimal convergence properties due to the complete implementation of the full Jacobian matrix.

A multiphase free energy system which implements formalisms such as the Kim-Kim-Suzuki multiphase method ties in with the modular free energy system and allows the construction of complex multiphase systems. We utilize this capability in MARMOT to provide material models for various fuel and structural material systems such as $\mathrm{UO}_{2} / \mathrm{Xenon}, \mathrm{USi}$, UZr, ZrH, and FeCr.

The tensor mechanics module contains the MOOSE components implementing a comprehensive set of solid mechanics models. It covers small strain linear elasticity, finite strain elasticity, and various plasticity models.

For MARMOT a modular mechanics system was developed based on MOOSE material classes. Strain, Eigenstrain, stress, and stiffness calculations are modular and can be combined as needed. The mechanics material system ties into the automatic differentiation system allowing for variable dependent properties (such as Eigenstrains and stiffnesses) to be constructed. Elastic energy density can be computed and used for coupling to the phase field equations.

New developments include a strain periodic boundary condition that enables the combined simulation of representative volume elements with periodic composition boundary conditions and periodic deformation. Applying the periodicity constraint to the strain enables the maximum amount of deformation degrees of freedom, such as arbitrary shear and volume non-conserving deformation.

MARMOT contains specific material models for nuclear applications. A new development for

modeling oxide fuel systems is a free energy based cluster dynamics system for the evolution of $\mathrm{UO}_{2}$ point defects and Xenon gas atom/defect complexes. 


\section{Stage 3 fission gas release modeling \\ 3.1 Introduction}

In the current implementation of the Simple Fission Gas Release (SiFGRS) model in BISON, the local fractional grain boundary fission gas coverage $F_{c}$ is tracked at each position in the fuel. When $F_{c}$ goes above a saturation coverage level, $F_{c, s a t}$, it is assumed that fission gas is immediately released such that $F_{c}=F_{c, s a t}=0.5$ is maintained. However, this assumption does not account for the need for a percolated pathway from that position in the fuel to a free surface to allow gas to escape. To address this need, BISON was modified to determine whether a percolated pathway exists before fission gas release is allowed.

\subsection{Modifications to BISON}

To determine whether a percolated pathway exists for fission gas release, the SiFGRS material was modified, and the user object PercolationUserObject and aux kernel PercolationAux were added. These are used in conjunction with existing objects as follows. The quantity $F_{c}-F_{c, s a t}$ is calculated as an aux variable. When $F_{c}-F_{c, s a t} \geq 0$, the grain boundaries in that region of the fuel are considered to be fully connected, and that region is checked to determine whether it connected to a free surface to allow gas release. The FeatureFloodCount user object is used to identify clusters of regions where $F_{c}-F_{c, s a t} \geq 0$. The PercolationUserObject checks which of these clusters contact a free surface where gas can be released, and PercolationAux sets an aux variable $P=1$ for regions that are part of a cluster and connected to a free surface. The modified SiFGRS material checks if $P=1$ in addition to $F_{c}-F_{c, s a t} \geq 0$ at each position to determine whether fission gas release is allowed.

\subsection{Initial Testing}

The modified BISON model of Stage 3 fission gas release was tested using a 2D axisymmetric simulation of a single $\mathrm{UO}_{2}$ fuel pellet. The pellet radius is $5.3 \mathrm{~mm}$ and its height is $14.95 \mathrm{~mm}$. A $24 \times 16$ mesh with second-order Lagrange elements was used. The heat generation rate due to fission and thermal conductivity are assumed to be constant, and a Dirichlet boundary condition of $T=673 \mathrm{~K}$ was set at the radial exterior surface $(r=5.3 \mathrm{~mm})$. No-flux boundary conditions were used at the top and bottom surface to simulate a pellet in the middle of the fuel rod and simplify conditions as much as possible. The modified SiFGRS model was used. Two cases were considered: Case 1, where fission gas can be released only at the radial exterior surface; and Case 2, where fission gas can be released at the top and bottom surfaces as well as the radial exterior surface.

The results at the end of the simulation time (1157 days) are shown for Case 1 and Case 2 in Figure 3. The pellet centerline is at left in each subfigure. In Case 1 (Figure 3a-3b), where the top and bottom surfaces are not free surfaces for gas release, a percolated pathway has not formed, and thus $P=0$ throughout and no gas has been released. One consequence is that $F_{c}>1$ for some regions, as shown in Figure 3b. Because $F_{c}$ is the fractional grain boundary coverage, this is an unphysical result, and the model will need to be modified to account for this situation. For Case 2 (Figure 3c-3d), where gas can also be released through the top and bottom of the pellet, $P=1$ for a portion of the pellet from early in simulation time, and thus gas can be released throughout most of the simulation time. As a result, $F_{c}$ is maintained at a maximum of 0.5 , as shown in Figure $3 \mathrm{~d}$. 


\subsection{Summary and Future Plans}

Modifications to BISON have been tested to account for the need for a percolated pathway to a free surface before allowing fission gas release. This required modifications to the SiFGRS model and the addition of the user object PercolationUserObject and aux kernel PercolationAux. In testing on a single $\mathrm{UO}_{2}$ fuel pellet, the modifications correctly enforced the need for a percolated pathway before allowing fission gas release. However, when sufficient fission gas is produced without a release pathway, the fractional grain boundary coverage can exceed 1 . Future plans are to address the buildup of fission gases by allowing the transfer of gas to adjacent regions as $F_{c}$ approaches 1 locally. Once this has been implemented and tested on the single pellet geometry, testing will move to more realistic geometries, and issues identified in those tests will be corrected. The Riso AN3 test case from the BISON assessment will allow the most thorough comparison between simulation results and experiment, as it is one of the few test cases where measured data on the amount of fission gas released to the fuel rod free volume is available. 


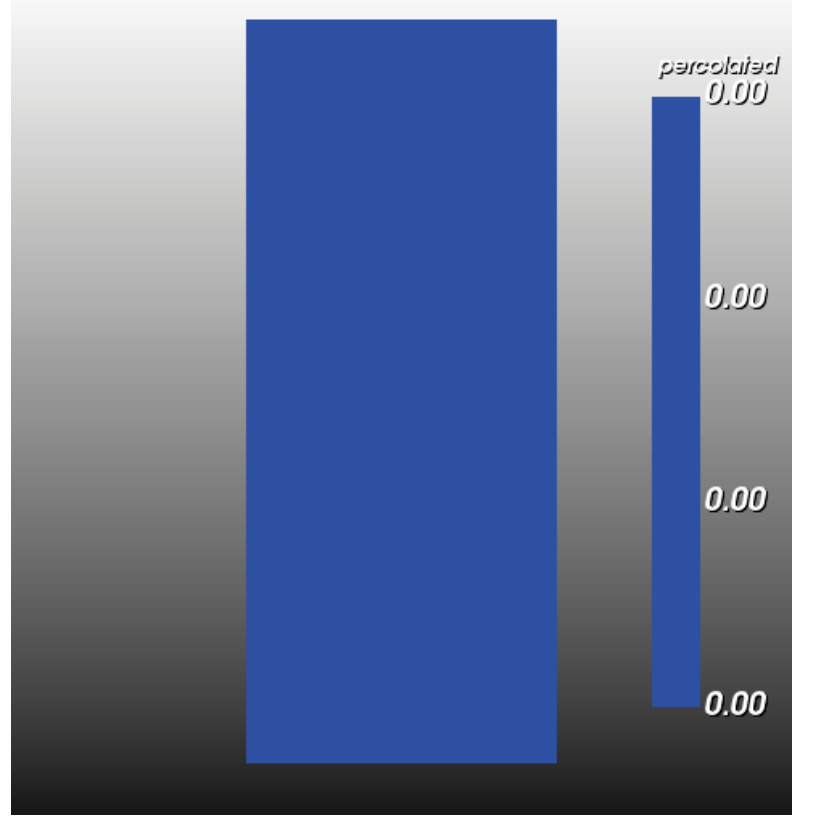

(a) Case 1, $P$

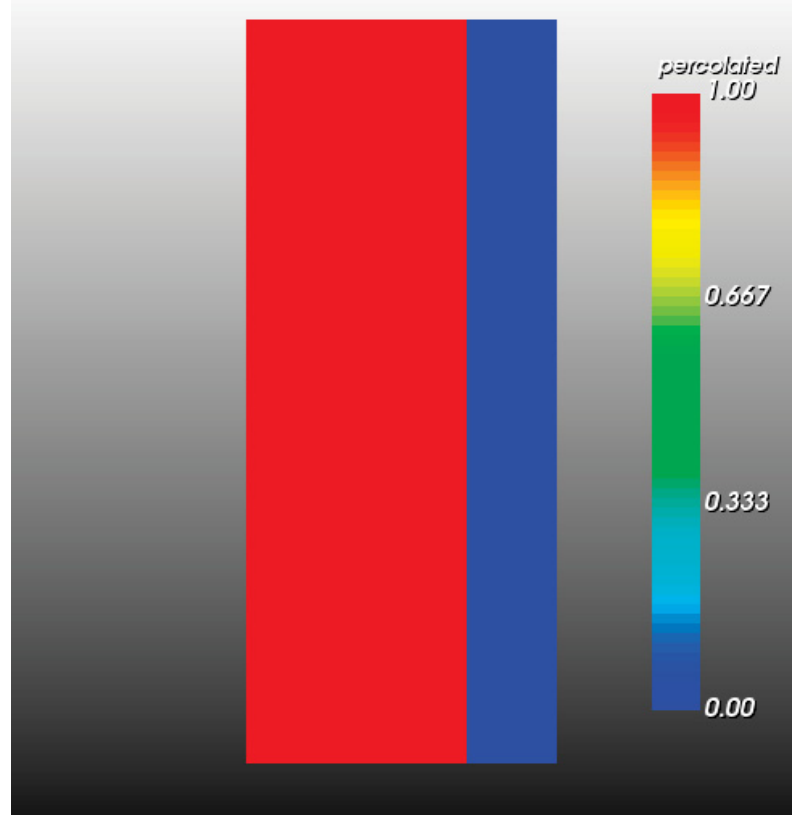

(c) Case 2, $P$

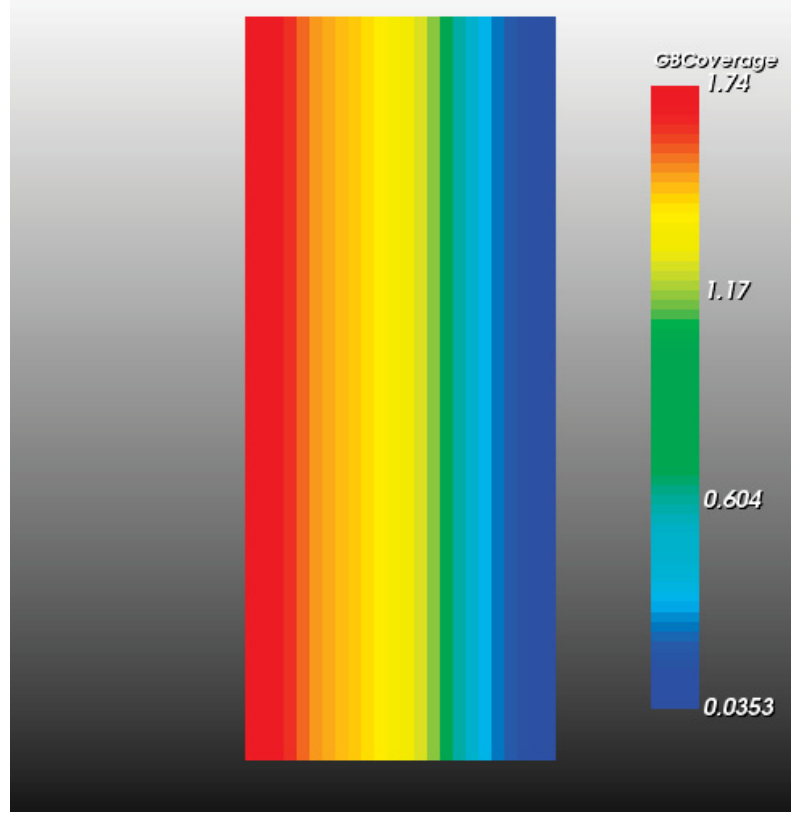

(b) Case $1, F_{c}$

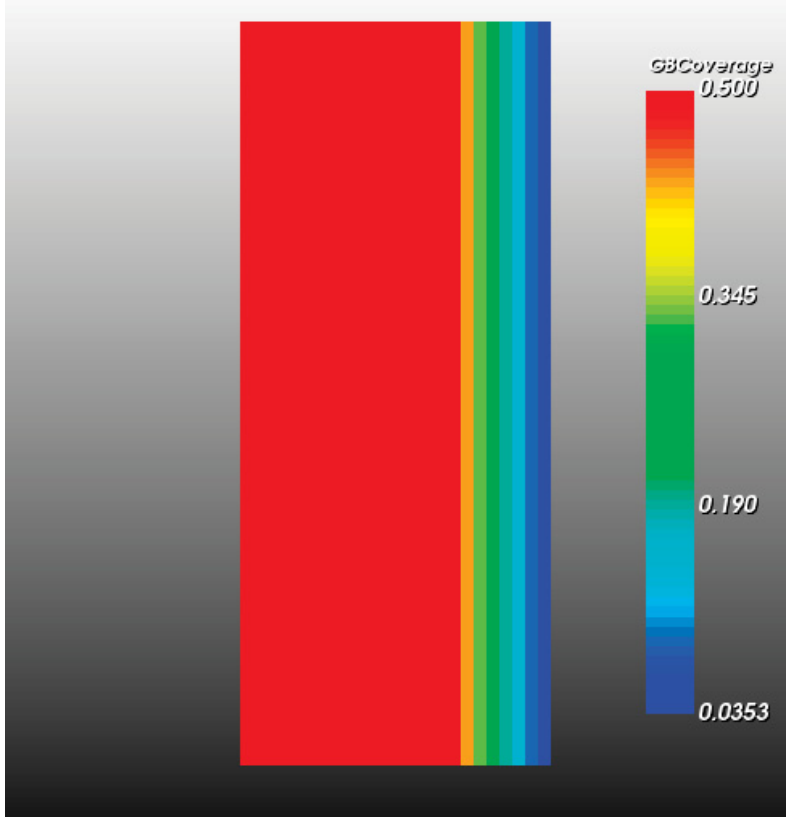

(d) Case $2, F_{c}$

Figure 3: 2D axisymmetric simulations of a single $\mathrm{UO}_{2}$ fuel pellet. Fuel pellet centerline is at left, radial exterior surface at right in each subfigure. In Case 1, fission gas can only escape through the radial exterior surface, so no percolated pathway exists, and $F_{c}>1$, which is unphysical. In Case 2, fission gas can also escape through the top and bottom, so percolation happens early in the simulation, and $F_{c}=0.5$ is maintained throughout. 


\section{MARMOT grain growth development}

All physical properties of $\mathrm{UO}_{2}$ are strongly dependent on the grain size. This is due to the prominent role of grain boundaries (GBs) in influencing material properties. Samples with smaller grain size have higher grain boundary area per unit volume. Furthermore, it was found out that the performance of $\mathrm{UO}_{2}$ under extreme conditions, such as irradiation, high temperature, high stresses, etc., is highly affected by the grain size. For instance, theory and experiments showed a significant reduction of fission-gas release and dimensional changes in $\mathrm{UO}_{2}$ fuel with increasing grain size. Therefore, investigating the kinetics of grain growth in uranium dioxide is important for understanding the performance of the main nuclear fuel in nuclear reactors. Pore drag, solute drag and anisotropy of GB energy may affect the overall grain growth rates in materials. Here we focus our attention on the effect of pore drag on the process of grain growth at the phase field level and the anisotropic GB energies in $\mathrm{UO}_{2}$ at the atomic level.

\subsection{Phase field model development in MARMOT considering pore dragging}

While a few phase field models of grain growth have been implemented in MARMOT, a model that accounts for the effect of mobile pores on the grain growth kinetics was lacking. We recently developed and implemented a such model in MARMOT. The model takes into account both the curvature-driven grain boundary motion and pore migration by surface diffusion. As such, the model accounts for the interaction between pore and grain boundary kinetics, which tends to retard the grain growth process. Our 2D and 3D simulations demonstrate that the model capture all possible pore-grain boundary interactions proposed in theoretical models. For high enough surface mobility, the pores move along with the migrating boundary as a quasi-rigid-body, albeit hindering its migration rate compared to the pore-free case. For less mobile pores, the migrating boundary can separate from the pores. For the pore-controlled grain growth kinetics, the model predicts a strong dependence of the growth rate on the number of pores, pore size, and surface diffusivity in agreement with theoretical models. An evolution equation for the grain size that includes these parameters was derived and showed to agree well with numerical solution. It shows a smooth transition from boundary-controlled kinetics to pore-controlled kinetics as the surface diffusivity decreases or the number of pores or their size increases. Further details are available in Ref. [6]. The evolution equation for the grain size developed here will be utilized in BISON to give accurate estimate for the grain size evolution. The effect of solute drag and anisotropy of grain boundary energy on grain growth will be investigated in future studies.

Two oral presentations were given about this work at conferences. The first is entitled "3D Phase Field Simulation of Grain Growth in Porous $\mathrm{UO}_{2}$ " and was presented at the TMS meeting in February 2016 in Nashville,TN. The second is entitled "Phase Field Modeling of Grain Growth in $\mathrm{UO}_{2} "$ and was presented at the MRS Spring meeting in April 2016 in Phoenix,AZ.

\subsection{Anisotropic grain boundary energies in $\mathrm{UO}_{2}$ \\ 4.2.1 Introduction}

In mesoscale simulations such as phase field simulations, microstructure features such as voids, precipitates, and GBs can be explicitly represented and individually tracked for their evolutions with time. For this purpose, the properties of each GB such as energy and mobility are needed as inputs and they are largely unknown for $\mathrm{UO}_{2}$. While energies [7] and mobilities [8] have been 
obtained by MD simulations for a few representative GBs, the same data are missing for the vast number of others. Furthermore, as in reality an infinite number of GBs may exist, in mesoscale modeling the properties are needed for all of them. Therefore, continuous models that can fully describe anisotropic GB properties are desired. In the present work, such a model is established for GB energies in $\mathrm{UO}_{2}$.

In FY15 and FY16 we extended the 5 dimensional GB model developed in Bulatov et al. [9] (referred to as the $5 \mathrm{D}$ model) to $\mathrm{UO}_{2}$, which is a ceramic with the fluorite crystal structure. The $5 \mathrm{D}$ model is constructed upon the 5 geometric degree-of-freedoms (DOFs) of GBs, usually represented by 3 DOFs for misorientation plus 2 DOFs for GB inclination. The 3 DOFs for misorientation are associated with a rotation axis $[h k l]$ (2 DOFs as only the direction is needed) and the corresponding angle of rotation $\xi$. Two other angles, $\eta$ and $\Phi$, together define the inclination of GB plane. The angle $\Phi$ is the angle between the rotation axis $[h k l]$ and the normal of the GB plane; it is 0 for pure twist GBs and $\pi / 2$ for pure tilt. The angle $\eta$ measures the deviation of a non-twist GB from a symmetric GB. It is 0 for symmetric tilt GBs (STGBs) by definition, and taken as 0 for twist GBs for convenience. An arbitrary GB can be fully defined (0D) by supplying all 5 DOFs, and specifying $N$ DOFs will define a $5-N$ dimensional (e.g., 3D if $\mathrm{N}=2$ ) subset of GBs. For instance, $\left[\begin{array}{lll}h & k & l\end{array}\right]$ GBs with the rotation axes being $\left[\begin{array}{lll}h & k & l\end{array}\right]$ refer to a $3 \mathrm{D}$ subset as the three angles are not defined.

The 5D model uses the above approach to define a GB and assumes that GB energy is a continuous function of the 5 geometric DOFs. Based on this assumption a closed-form expression has been proposed and fitted for $\mathrm{Al}, \mathrm{Au}, \mathrm{Cu}$ and $\mathrm{Ni}$ [9]. The model contains 43 parameters, one specifying the scale of GB energy and the other 42 governing the shape of the functions. For the latter 42 parameters, 27 of them are shared by all four fcc metals, and the remaining 15 can be scaled from one metal to another by a single, material-specific parameter. Therefore, in the entire model there are only two material-specific parameters, suggesting that indeed GB energy can be described by the symmetry of fcc crystal using the 5 geometric DOFs. However, the exact functional form defining how GB energy depends on the 5 DOFs is uncertain, and tremendous data may be required to fit the model within a $5 \mathrm{D}$ space. To overcome these difficulties, another assumption was made that the 5D GB energy surface can be constructed by interpolation of special, lower dimensional subsets where GB energies are local minimums. Following this assumption, a hierarchical interpolation approach was used and the amount of data needed for interpolation was substantially reduced as the majority of the fitting was performed for lower-dimensional subsets only.

Practically, in the 5D model the energy of an arbitrary GB can be interpolated as a weighted average of simulated GBs that fall into 3D subsets denoted by their rotation axes, $[h k l]$ GBs. Three such 3D subsets have been chosen and they are the $<100>,<110\rangle$ and $<111>$ subsets. Note that in fcc or cubic crystals, $<h k l>$ denotes a family of $[h k l]$ axis with $h, k$, and $l$ interchangeable, and they can be of either positive and negative signs, giving 24 rotation-reflections. Such symmetry exists for fluorite ceramics as well. The energy surface of each $<h k l>$ subset can be obtained by interpolation of $1 \mathrm{D}$ twist GB subsets $(\Phi=\eta=0)$ and $2 \mathrm{D}$ tilt GB subsets $(\Phi=\pi / 2)$ with the same rotation axis. The energy surfaces for the $2 \mathrm{D}$ tilt GB subsets are constructed by linear interpolation of 1D STGBs in the same 2D subset. Finally, for 1D GB subsets such as $<h k l>$ twist and $<h k l>\operatorname{STGBs}(\Phi=\pi / 2 ; \eta=0)$, the only undefined DOF is the rotation angle $\xi$. For these two cases, the Read-Shockley-Wolf (RSW) function is used. Based on crystal geometry, Read and Shockley [10] proposed a dislocation-based model for low-angle tilt GBs in cubic crystals, which was further extended by Wolf [11] to cover high-angle tilt and twist GBs as well. We note that the RSW function has also been used for the interpolations from 3D subsets to 5D GBs and from 1D STGBs to 2D pure tilt GBs in the $<110>3 \mathrm{D}$ subset. However, the physical justification 


\section{$\gamma$-surface of GB energy $\left(\mathrm{J} / \mathrm{m}^{2}\right)$}
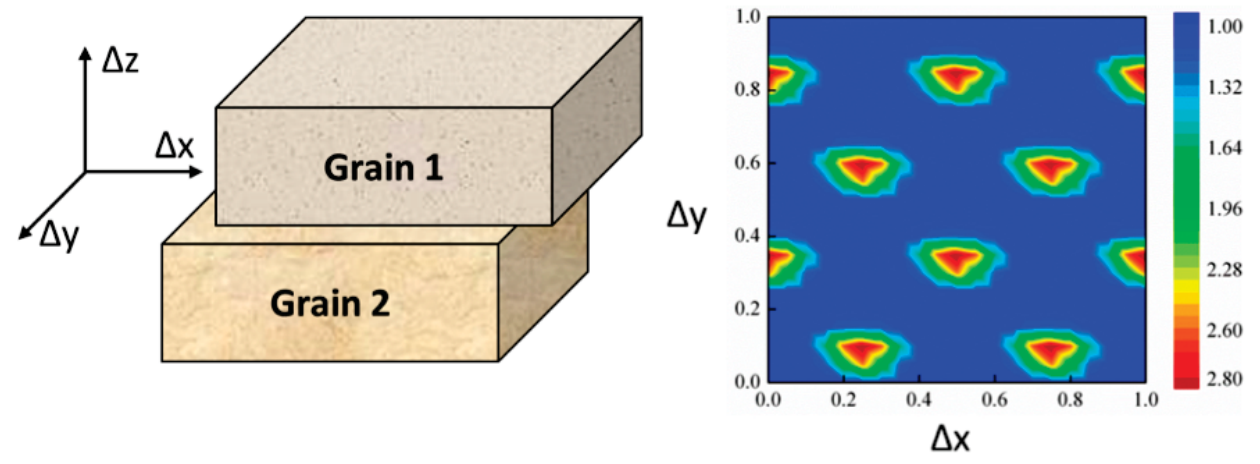

Figure 4: Schematic of the bi-crystal model and the three microscopic translational DOFs (left) and the $\gamma$-surface for the $<110>\Sigma 3$ STGB (right).

for using the RSW function in these two cases is not as clear as for the 1D twist and STGBs. We use least-square fitting to determine the parameters.

To fit the needed parameters in the 5D model requires large amount of data on the GB energies in $\mathrm{UO}_{2}$, which unfortunately do not exist. Here, we use MD simulations to calculate GB energies needed to fit the 5D model. We use the LAMMPS simulation package [12] developed at Sandia National Laboratory for our simulations. The interatomic interaction is described by the Basak potential [13] which has been shown to reproduce well the structure of the $<100>\Sigma 5$ STGBs in reference to DFT calculations. To calculate GB energies, here we follow the $\gamma$-surface method (see Fig.4) used in Nerikar et al..[14]. In addition to the $0 \mathrm{~K}$ calculations, annealing of GBs at $800 \mathrm{~K}$ was also carried out, followed by a minimization to obtained the GB energy at $0 \mathrm{~K}$. The calculation is done for $171 \mathrm{GBs}$, of which 158 are used for fitting and the rest for verification. These include symmetrical tilt grain boundaries (STGBs), pure twist, asymmetrical tilt, and random grain boundaries for generalness.

\subsubsection{Results}

In Fig.5 the fitting results for all six 1D subsets are shown. Since the MD results are approximate due to the use of an empirical potential and finite simulation cell sizes, a $5 \%$ uncertainty is added to all MD data. As can be seen from the figure, the results obtained from MD simulations with the Basak potential can be fitted well by the RSW function. For most 1D subsets, more than one RSW functions are used, depending on the number of cusps in the rotational space. Here, the number of cusps and their positions in term of rotation angle are taken as the same as in Bulatov et al. for fcc metals. [9] It is impressive to see that the cusps for fcc metals are also cusps for $\mathrm{UO}_{2}$ in the same 1D subset, due of the similarity of fcc and fluorite structures, indicating the dominant role of crystal geometry on GB energy. However, the cusps in $\mathrm{UO}_{2}$ are usually not as deep as in fcc metals. It is also observed that for most cases, the annealing at $800 \mathrm{~K}$ can relax the GB structure further and leads to a lower GB energy.

In $\mathrm{UO}_{2}$, it is found that the $<100>$ twist GBs has the lowest energies compared to the other two twist subsets. At high angles the GB energy is about $1.0 \mathrm{~J} / \mathrm{m}^{2}$ for $<100>$ twist GBs, while it is about $1.4 \mathrm{~J} / \mathrm{m}^{2}$ for $<111>$ twist, and around $1.9 \mathrm{~J} / \mathrm{m}^{2}$ for $<110>$ twist. This trend is different from that in fcc metals, where the $<111>$ twist GBs have much lower energies than the $<100>$ and 

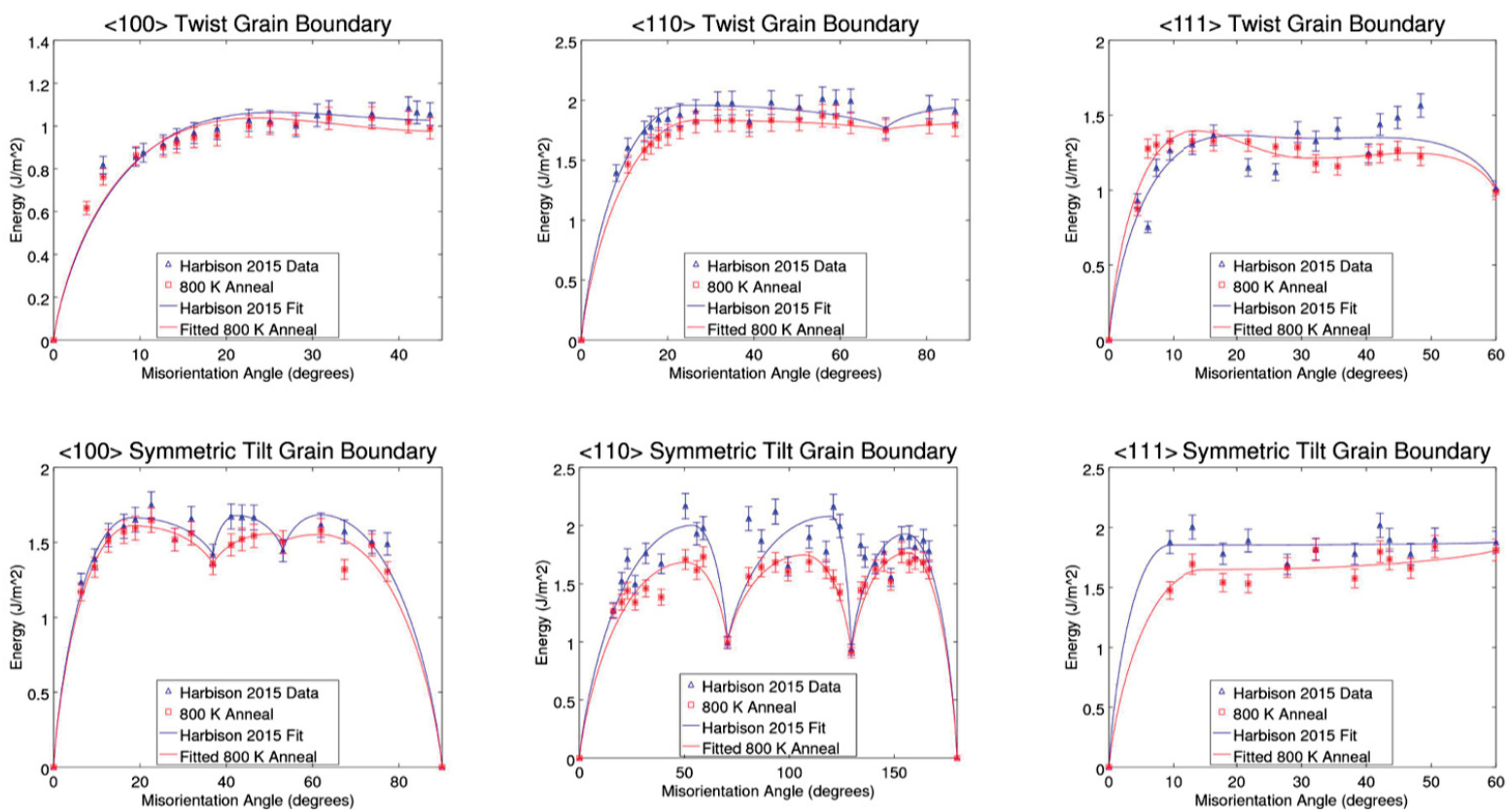

Figure 5: GB energy $\left(\mathrm{J} / \mathrm{m}^{2}\right)$ as a function of rotation angle for (a) $<100>$, (b) $<110>$ and (c) $<111>$ twist GBs, and (d) $<100>$, (e) $<110>$ and (f) $<111>$ STGBs. The dots are obtained by MD simulations, and the curves are fitted using RSW functions. Note that for each 1D subset, the RSW function may be used different times with different numbers of fitting parameters (shown as insets in the figures).
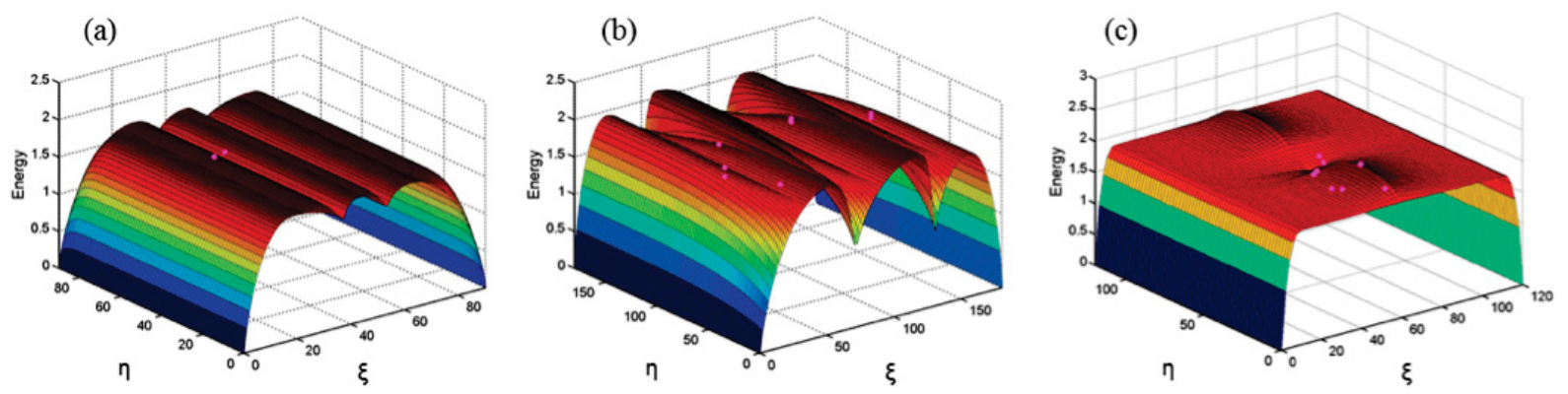

Figure 6: GB energy $\left(\mathrm{J} / \mathrm{m}^{2}\right)$ surfaces for $2 \mathrm{D}(\mathrm{a})<100>$, (b) $<110>$ and $(\mathrm{c})<111>$ tilt subsets as functions of angles $\xi$ and $\eta$, in units of degrees. The pink dots are MD results (in addition to those used in 1D subsets) used for fitting; some of them are hidden below the surface.

$<110>$ twist GBs at similar rotation angles. In fcc crystals, $\{111\}$ plane is the most closed-packed plane with the largest inter-plane distance. Therefore it is reasonable for GBs on $\{111\}$ planes to have lower energies than those on other planes. In $\mathrm{UO}_{2}$, U-ions follow fcc structure, and O-ions are of sc structure in which $\{100\}$ is the most closed-packed plane. A GB in $\mathrm{UO}_{2}$ on a $\{111\}$ plane will cut or distort all first nearest neighbor $\mathrm{O}-\mathrm{O}$ bonds along $<100\rangle$, leading to high $\mathrm{GB}$ energies. Furthermore, in $\mathrm{UO}_{2}\{111\}$ planes have a tri-layer structure, with one $\mathrm{U}$ sub-layer sandwiched by two O sub-layers. A GB on a $\{111\}$ plane destroys the stacking periodicity along $\langle 111\rangle$, and thus the relative positions of $\mathrm{O}$ ions in two neighboring $\mathrm{O}$ sub-layers. As all $\mathrm{O}$ sub-layers are negatively charged, a strong energy penalty can be induced, leading to high GB energies. 


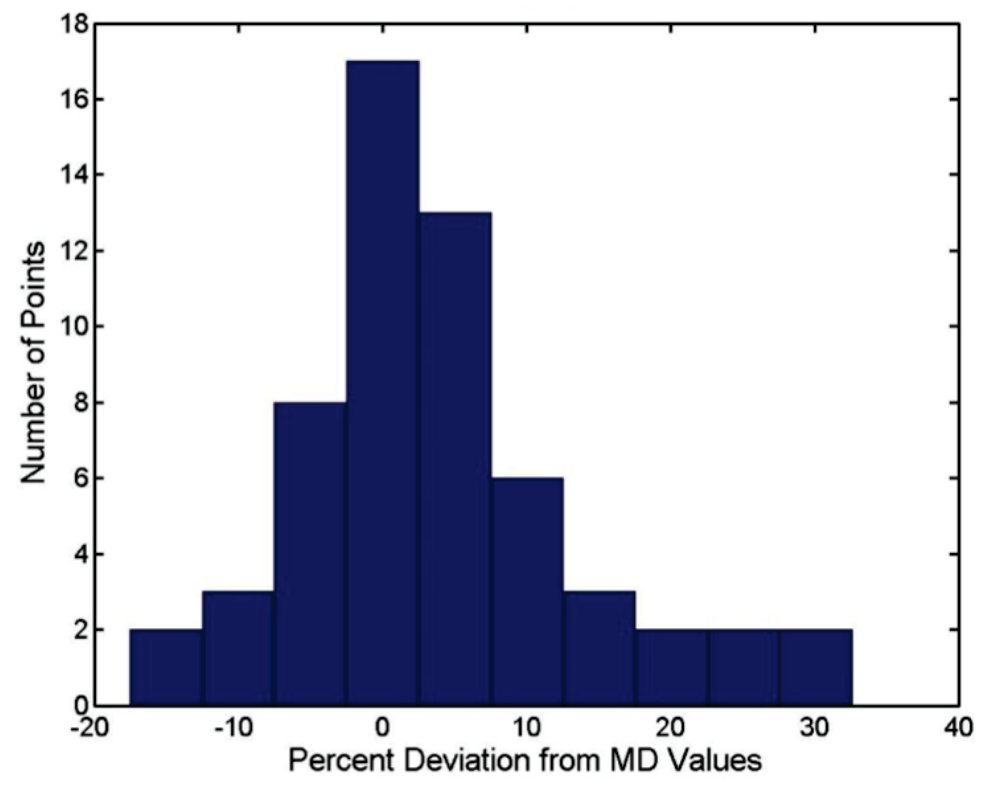

Figure 7: Statistics of fitting errors in term of percent deviation from MD value.

The fitted 1D STGB subsets are used to construct the 2D general tilt subsets with the same rotation axes. MD results for $2 \mathrm{D}$ grain boundaries are also needed for this purpose. In Fig.6, the fitting results for 2D grain boundaries are shown. Here, MD results from 0K without annealing are used. New fitting using the annealed data is currently going on. For $<100>$ and $<110>$ tilt subsets, the shapes of the GB energy surfaces are similar to those of fcc metals for the same subsets, but with much higher values. For the $<111>$ tilt subset, for most $\eta$ the GB energies reach a peak for $\mathrm{UO}_{2}$ at the rotation angle $\xi=60^{\circ}$ rather than a cusp as for fcc metals. As shown in Fig.2(f), the energy of the $1 \mathrm{D}<111>$ STGB subsets reaches a peak at $\xi=60^{\circ}$, so does that of the $2 \mathrm{D}<111>$ tilt subset.

The 3D and 5D fitting uses the 2D subsets and new MD data on random GBs. These results are hard to visualize for the high dimensions. The fitting has been done using MD results obtained at $0 \mathrm{~K}$ without annealing. Again, new fitting using the annealed data is in progress. Overall, the 5D model can fit all MD results well, with an the fitting error (deviation of the fitted values from MD results in percent) is within $10 \%$ for most GBs, as shown in Fig. 7.

The $5 \mathrm{D}$ model is expected to be able to predict the energy of any arbitrary GB so that the GB anisotropy can be fully considered. Although the model has been shown to fit well with MD data, it is also desired to check the predictivity. For this purpose, we have used MD simulations to calculate the energies of GBs in the $1 \mathrm{D}<210>$ twist subset. The MD results are compared with the model prediction in Fig. 8. In the prediction the 43 parameters in In Fig. 9 are used. Again, we note that this set of parameter is obtained using MD results at $0 \mathrm{~K}$ without annealing. They will be updated once the fitting using annealed results is finished. Overall, the MD results compare well with those given by the theoretical model. It is interesting to see that the $5 \mathrm{D}$ model captures the trends at the low angle region and the region close to $180^{\circ}$, and also the cusp at about $130^{\circ}$. The agreement between MD and the model prediction is impressive as these MD results are not included in the fitting procedure. 

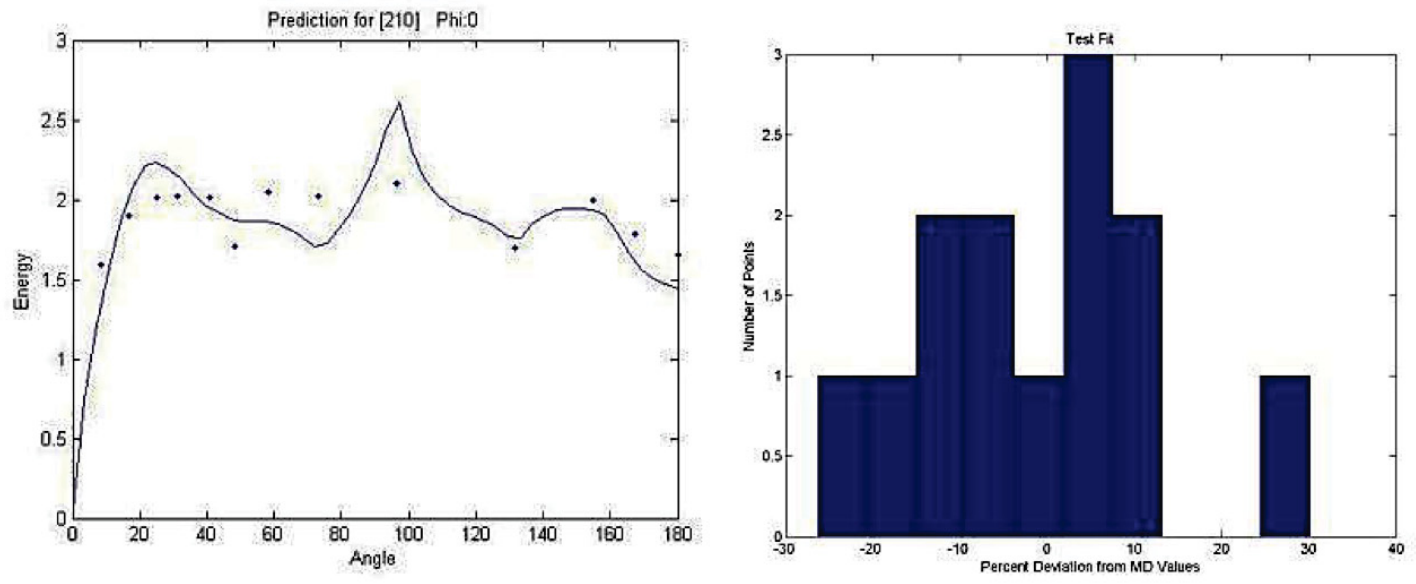

Figure 8: GB energy $\left(\mathrm{J} / \mathrm{m}^{2}\right)$ as a function of rotation angle for the $<210>$ twist subset. The dots are from MD simulations; the curve is predicted by the $5 \mathrm{D}$ model. On the right the prediction error in reference to the MD results is plotted.

\subsubsection{Implementation in MARMOT}

The above fitted 5D model has been implemented into MARMOT. In the phase field method, each grain in a polycrystalline solid is represented by an order parameter, with the corresponding orientation specified by three Euler angles [15]. The GB plane is given by the gradients of the order parameters for the two grains across a GB. So the $5 \mathrm{D}$ space is covered by 3 DOFs from Euler angles and 2 DOFs from the gradients of order parameters. Note that the summation of the two order parameters across a GB gives 1, so their gradients are of the same direction but with opposite signs, giving 2 DOFs in total. Again, only the normal of the gradient is needed. Therefore, to obtain the energy of a GB defined in a phase field model using the 5D model, two steps are needed: (1) rotate the GB plane given by the gradients of order parameters to the $z$ axis; and (2) calculate the rotation matrices for the two grains. In Fig.10, the anisotropic GB energy calculate in MARMOT for a polycrystalline sample is shown the demonstrate the capability. Current, the model is being tested for grain growth simulations. In the future, it will be used to investigate the effect of anisotropic GB energy on grain growth and gas bubble formation. 


\begin{tabular}{|c|c|c|c|c|c|c|c|}
\hline No. & Name & Description & Value & No. & Name & Description & Value \\
\hline 1 & $\varepsilon_{R G B}$ & $\begin{array}{c}\text { Energy Scaling } \\
\text { Factor }\end{array}$ & $\begin{array}{c}1.615789 \\
\mathrm{~J} / \mathrm{m}^{2}\end{array}$ & 23 & $\varepsilon_{110}^{t w i s t, \max }$ & 110 Twist Peak Energy & 1.211934 \\
\hline 2 & $d_{100}^{\max }$ & 100 Max Distance & 0.405 & 24 & $\varepsilon_{110}^{t w i s t, \Sigma 3}$ & 110 Twist $\Sigma 3$ & 1.092599 \\
\hline 3 & $d_{110}^{\max }$ & 110 Max Distance & 0.739 & 25 & $\varepsilon_{110}^{t w i s t, 90}$ & $\begin{array}{c}110 \text { Twist } 90 \text { Degree } \\
\text { Energy }\end{array}$ & 1.20006 \\
\hline 4 & $d_{111}^{\max }$ & 111 Max Distance & 0.352 & 26 & $\alpha_{110}^{a t g b}$ & $\begin{array}{l}110 \text { Asymmetric Tilt } \\
\text { Shape Factor }\end{array}$ & 1.0 \\
\hline 5 & $w_{100}^{0}$ & 100 Weight & 26.2 & 27 & $\varepsilon_{110}^{s t g b, \max 3}$ & $\begin{array}{l}110 \text { Symmetric Tilt } \\
\text { Third Peak Energy }\end{array}$ & 1.238721 \\
\hline 6 & $w_{110}^{0}$ & 110 Weight & 0.65 & 28 & $\varepsilon_{t w i n}$ & 110 Symmetric Tilt $\Sigma 3$ & 0.612704 \\
\hline 7 & $w_{111}^{0}$ & 111 Weight & 6.1 & 29 & $\varepsilon_{110}^{s t g b, \max 2}$ & $\begin{array}{l}110 \text { Symmetric Tilt } \\
\text { Second Peak Energy }\end{array}$ & 1.287202 \\
\hline 8 & $p_{100}^{1}$ & $\begin{array}{c}100 \text { Tilt/Twist Mix } \\
\text { Power Law }\end{array}$ & 0.363719 & 30 & $\varepsilon_{110}^{s t g b, \Sigma 11}$ & 110 Symmetric Tilt $\Sigma 11$ & 0.576808 \\
\hline 9 & $p_{100}^{2}$ & $\begin{array}{c}100 \text { Tilt/Twist Mix } \\
\text { Power Law }\end{array}$ & 0.000141 & 31 & $\varepsilon_{110}^{s t g b, \max 1}$ & $\begin{array}{l}110 \text { Symmetric Tilt } \\
\text { First Peak Energy }\end{array}$ & 1.193228 \\
\hline 10 & $\varepsilon_{100}^{t w i s t, \max }$ & $\begin{array}{c}\text { Maximum } 100 \\
\text { Twist Energy }\end{array}$ & 0.635392 & 32 & $\xi_{110}^{s t g b, \max 3}$ & $\begin{array}{l}110 \text { Symmetric Tilt } \\
\text { Third Peak Angle }\end{array}$ & 0.944648 \\
\hline 11 & $\alpha_{100}^{t w i s t}$ & $\begin{array}{l}100 \text { Twist Shape } \\
\text { Factor }\end{array}$ & 1.320973 & 33 & $\xi_{110}^{s t g b, \max 2}$ & $\begin{array}{l}110 \text { Symmetric Tilt } \\
\text { Second Peak Angle }\end{array}$ & 2.09343 \\
\hline 12 & $p$ & $\begin{array}{l}100 \text { Asymmetric } \\
\text { Tilt Interpolation }\end{array}$ & 34.1 & 34 & $\xi_{110}^{s t g b, \max 1}$ & $\begin{array}{l}110 \text { Symmetric Tilt } \\
\text { First Peak Angle }\end{array}$ & 2.717258 \\
\hline 13 & $\varepsilon_{100}^{s \operatorname{stg}, \max 1}$ & $\begin{array}{l}100 \text { Symmetric Tilt } \\
\text { First Peak Energy }\end{array}$ & 1.030398 & 35 & $\alpha$ & $\begin{array}{l}111 \text { Tilt-Twist } \\
\text { Interpolation }\end{array}$ & 0.606289 \\
\hline 14 & $\varepsilon_{100}^{s t g b, \Sigma 5,1}$ & $\begin{array}{l}100 \text { Symmetric Tilt } \\
\text { First } \Sigma 5 \text { Energy }\end{array}$ & 0.876971 & 36 & $\alpha_{111}^{t w i s t}$ & 111 Twist Shape Factor & 1.197468 \\
\hline 15 & $\varepsilon_{100}^{s t g b, \max 2}$ & $\begin{array}{l}100 \text { Symmetric Tilt } \\
\text { Second Peak Energy }\end{array}$ & 1.038988 & 37 & $\xi_{111}^{\text {twist,max }}$ & 111 Twist Peak Angle & 0.546169 \\
\hline 16 & $\varepsilon_{100}^{s \operatorname{stg}, \Sigma 5,2}$ & $\begin{array}{l}100 \text { Symmetric Tilt } \\
\text { Second } \Sigma 5 \text { Energy }\end{array}$ & 0.893062 & 38 & $\varepsilon_{111}^{t w i s t, \max }$ & 111 Twist Peak Energy & 0.832776 \\
\hline 17 & $\varepsilon_{100}^{s t g b, \Sigma 17}$ & $\begin{array}{l}100 \text { Symmetric Tilt } \\
\text { \&17 Energy }\end{array}$ & 1.041893 & 39 & $\xi_{111}^{\text {tilt } \text { max }}$ & $\begin{array}{l}111 \text { Symmetric Tilt } \\
\text { Peak Angle }\end{array}$ & 0.164954 \\
\hline 18 & $\xi_{100}^{\text {stgb,max } 1}$ & $\begin{array}{l}100 \text { Symmetric Tilt } \\
\text { First Peak Angle }\end{array}$ & 0.312093 & 40 & $\varepsilon_{111}^{s t g b, \max }$ & $\begin{array}{c}111 \text { Symmetric Tilt } \\
\text { Max Energy }\end{array}$ & 1.146105 \\
\hline 19 & $\xi_{100}^{s t g b, \max 2}$ & $\begin{array}{l}100 \text { Symmetric Tilt } \\
\text { Second Peak Angle }\end{array}$ & 0.739168 & 41 & $\varepsilon_{111}^{s t g b, \Sigma 3}$ & 111 Symmetric Tilt $\Sigma 3$ & 1.159805 \\
\hline 20 & $p_{110}^{1}$ & $\begin{array}{c}110 \text { Tilt/Twist Mix } \\
\text { Power Law }\end{array}$ & 0.154383 & 42 & $\varepsilon_{111}^{s t g b, \Sigma 3, \max }$ & $\begin{array}{l}111 \text { Asymmetric Tilt } \\
\text { Symmetry Point Energy }\end{array}$ & 1.330302 \\
\hline 21 & $p_{110}^{2}$ & $\begin{array}{c}110 \text { Tilt/Twist Mix } \\
\text { Power Law }\end{array}$ & 0.905391 & 43 & $\lambda$ & $\begin{array}{l}111 \text { Asymmetric Tilt } \\
\text { Scale Factor }\end{array}$ & 3.730652 \\
\hline 22 & $\xi_{110}^{t w i s t, \max }$ & $\begin{array}{c}110 \text { Twist Peak } \\
\text { Angle }\end{array}$ & 0.422022 & & & & \\
\hline
\end{tabular}

Figure 9: Parameters used in the 5D model for $\mathrm{UO}_{2}$. 


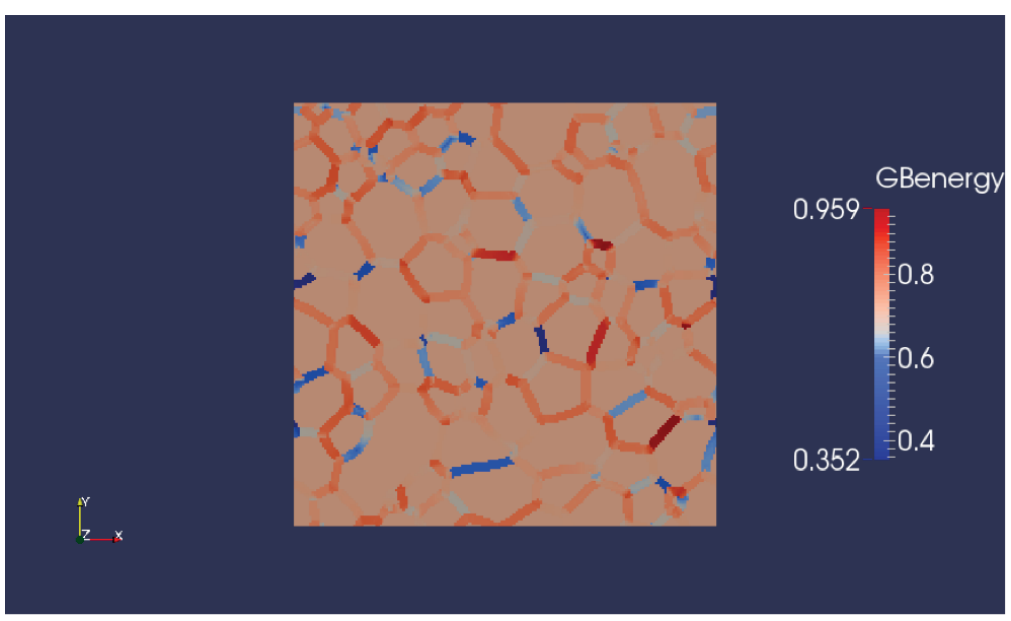

Figure 10: Relative GB energy $\left(\mathrm{J} / \mathrm{m}^{2}\right)$ for a polycrystalline sample calculated in MARMOT using the $5 \mathrm{D}$ model 


\section{High burn-up structure formation}

$\mathrm{UO}_{2}$ and other nuclear fuels develop a unique microstructure under irradiation usually known as the High Burn-up Structure (HBS). In this HBS, the as-fabricated microstructure transforms into a much finer one with a grain size that is one order of magnitude less than the initial grain size. The reduction of the strain energy-due to point defects and dislocations formed during irradiation-by forming new defect-free subgrains compensates for the excess energy required for creating new grain boundaries. The HBS is also a very porous structure, where gas bubbles usually decorate the grain boundaries of the fine, newly formed subgrains. It was shown that the HBS formation alters the mechanical and thermal properties of nuclear fuels. It also changes the swelling and gas release rates and hence affects the fuel integrity and performance. Therefore, investigating the formation and evolution of the HBS in nuclear fuels is of paramount importance for improving the reactor performance and safety.

Two main mechanisms for the HBS formation were proposed in literature. The first is called polygonization (grain subdivision). Polygonization is the subdivision of the original grains based on the reorganization of dislocations into sub-boundary domains. The other is recrystallization, which is characterized by the formation of subgrains and their subsequent growth into stable recrystallized grains. We focus here on the latter mechanism.

A quantitative phase field model of irradiation-induced recrystallization was developed and implemented in MARMOT. The model adds the stored energy contribution, associated with dislocations formed under irradiation, to the regular grain growth model to account for the formation

and subsequent growth of recrystallized grains. Stored energy kernel, action and Material class were added to MARMOT to facilitate the implementation of the model. The model predicts a threshold dislocation density for the formation of $\mathrm{HBS}$ in $\mathrm{UO}_{2}$ in agreement with theoretical predictions and experiments. Further details are available in Ref. [16]. The formation and growth of gas bubbles in the HBS will be included later. 


\section{Creep and fracture model development in polycrystalline solids}

The microstructure of $\mathrm{UO}_{2}$ fuels undergoes significant evolution during operation, which affects their subsequent thermo-mechanical-physical properties. Resolving the dependencies of the properties on the state of the microstructure and the history of evolution is important to obtain accurate prediction. One approach to develop microstructure-property correlation is through empirical models obtained from nano/micro-scale experiments. However, this approach can be expensive, time-consuming and in cases limited in applicability to the experimental conditions. The alternative approach is to develop experimentally validated physics-based models at different length scales that can then finally be linked to homogenized engineering scale laws. Following the second approach, a polycrystalline creep model has been developed in NEAMS and discussed in this section. Furthermore, the performance of a phase-field elasto-plastic fracture model developed in FY14 and 15, is compared with X-FEM, a sharp-interface method for fracture available in MOOSE.

\subsection{Polycrystalline creep model}

The knocking out of atoms during irradiation leads to the formation of interstitials and vacancies. The vacancies can diffuse and cluster together to form voids causing the material to swell. Also, the concerted motion of the vacancies can cause distortional changes that results in time dependent material creeping. Such vacancy diffusion driven creep can also occur under non-irradiated conditions at low stress levels. At low temperature and stress levels, vacancies preferably diffuse along grain boundaries and the creep rate can be represented by the Coble-creep law

$$
\frac{d \epsilon}{d t}=\frac{\sigma}{d^{3}} D_{g b} \exp \left(-\frac{Q}{R T}\right)
$$

where $\sigma$ is the stress, $d$ is the average grain-size, $D_{g b}$ is the grain boundary diffusivity, $Q$ is the activation energy, $R$ is the gas constant and $T$ is the temperature. At higher temperatures and stress levels, bulk diffusion of vacancies is the primary mechanism and is designated by the NabarroHerring $(\mathrm{N}-\mathrm{H})$ creep law following

$$
\frac{d \epsilon}{d t}=\frac{\sigma}{d^{2}} D_{b} \exp \left(-\frac{Q}{R T}\right)
$$

where $D_{b}$ is the bulk vacancy diffusivity. As can be observed from both the equations, the microstructural feature viz. grain-size has a significant influence on the creep rate. However, for spatially varying microstructures or in the presence of other defects, typical under irradiation, the utilization of these simple models may not be practical. Moreover, stress and temperature gradients may necessitate coupling of vacancy diffusion mechanism with other deformation processes. Thus, though the above mentioned creep-rate laws are applicable under well defined homogeneous conditions, their predictions can be erroneous for realistic scenarios the fuels experience. To address this limitation, polycrystalline-scale creep model is developed in this work. The length-scale of the model facilitates incorporation of the irradiation process, other defects, thermo-mechanical and microstructural (grain-size) gradients, and, coupling to other deformation processes.

The model developed here is based on [17]. The evolution of vacancy concentration is based on the substitutional motion of atoms and is represented by

$$
\dot{c}_{v}=-\frac{\partial j_{i}}{\partial x_{i}}
$$


where $c_{v}$ is the vacancy concentration, $j_{i}$ are the flux components along directions $\mathrm{i}=1,2$ and 3 . The vacancy flux evolves following the Onsager's relation

$$
j_{i}=-L_{i j} \frac{\partial \mu}{\partial x_{j}}
$$

where $L_{i j}$ is the mobility and $\mu$ is the chemical potential. The chemical potential is related to the free-energy of the system $(\psi)$ that has both a chemical $\left(\psi_{c}\right)$ and a mechanical $\left(\psi_{m}\right)$ component. The mechanical contribution is related to the elastic strain energy following

$$
\psi_{m}=\frac{1}{2} \epsilon_{i j}^{e} C_{i j k l} \epsilon_{k l}^{e}
$$

where $C_{i j k l}$ is the $4^{t h}$ order elasticity tensor and the elastic strain $\left(\epsilon_{i j}^{e}\right)$ can be represented by

$$
\epsilon_{i j}^{e}=\epsilon_{i j}-\epsilon_{i j}^{v}-\epsilon_{i j}^{c r}
$$

In Eq.6, $\epsilon_{i j}$ is the total strain, $\epsilon_{i j}^{c r}$ is the inelastic creep strain and $\epsilon_{i j}^{v}$ is the relaxation strain due to vacancies. A concentration dependent relaxation strain is proposed and follows

$$
\epsilon_{i j}^{v}=\eta_{i j} c_{v}
$$

where $\eta_{i j}$ is the relaxation tensor. The chemical energy is described using

$$
\psi_{c}=\frac{E_{f}}{\Omega_{0}} c_{v}+\frac{R T}{\Omega_{0}}\left(c_{v} \log c_{v}+\left(1-c_{v}\right) \log \left(1-c_{v}\right)\right)
$$

where the first term is related to the formation energy of vacancies and the second term represents the entropy of mixing. In Eq.8, $E_{f}$ is the formation energy, $\Omega_{0}$ is the molar volume, $R$ is the gas constant and $T$ is the absolute temperature. From the total free energy, the stress can be obtained as

$$
\sigma_{i j}=\frac{\partial \psi}{\partial \epsilon_{i j}^{e}}=C_{i j k l} \epsilon_{k l}^{e}
$$

and the chemical potential as

$$
\mu=\frac{\partial \psi}{\partial c_{v}}=\frac{E_{f}}{\Omega_{0}}+\frac{R T}{\Omega_{0}} \log \left(\frac{c_{v}}{1-c_{v}}\right)-\eta_{i j} \sigma_{i j}
$$

In this model, the vacancy diffusivity and relaxation tensor on the grain boundary (GB) is considered to be anisotropic, while the bulk behavior is isotropic. Since, the GBs act as vacancy source/sink, diffusion is allowed only along the non-normal direction on GBs. This is enforced through the diffusivity tensor $\left(D_{i j}\right)$ following

$$
D_{i j}=(1-\phi) D_{B} \delta_{i j}+\phi D_{G B}\left(\delta_{i j}-n_{i} n_{j}\right)
$$

where $\phi$ is the GB phase parameter, which is 0 in the bulk and 1 on GB; $D_{B}$ and $D_{G B}$ are the bulk and GB diffusivities, respectively and $n_{i}$ is the GB normal. The mobility is then represented as

$$
L_{i j}=\frac{\Omega_{0}}{R T} c_{v}\left(1-c_{v}\right) D_{i j}
$$

The relaxation tensor is modified accordingly, 


$$
\eta_{i j}=(1-\phi) \eta_{B} \delta_{i j}+\phi \eta_{G B} n_{i} n_{j}
$$

wherein the relaxation due to the presence of vacancies is assumed only along the GB normal direction.

In the model, the creep strain due to vacancy diffusion is obtained from the material velocity due to their directional substitution with vacancies. Assuming, that the number of substitutional sites remain approximately constant, the creep strain rate is derived as

$$
\dot{\epsilon}_{i j}^{c r}=-\left(\frac{1}{2}\left(\frac{\partial j_{i}}{\partial x_{j}}+\frac{\partial j_{j}}{\partial x_{i}}\right)-\frac{1}{3} \frac{\partial j_{k}}{\partial x_{k}} \delta_{i j}\right)(1-\phi)+\phi \eta_{G B} K_{s v}\left(c_{v}-c_{v}^{e q}\right) n_{i} n_{j}
$$

where $K_{s v}$ is a constant. The first term dominates in the bulk, whereas the second term is due to the addition or deletion of lattice sites in the GB region. The overall form maintains the isochoric nature of the creep process.

The model is implemented in MOOSE, where the stress equilibrium equation is solved in conjunction with the vacancy evolution equation. The following weak-forms have been implemented for Eqs.3, 4 and 10 as shown below:

$$
\int_{V} \delta c_{v} \dot{c_{v}} d V+\int_{V} \frac{\partial \delta c_{v}}{\partial x_{i}} L_{i j} \frac{\partial \mu}{\partial x_{j}} d V-\int_{S} \delta c_{v} L_{i j} \frac{\partial \mu}{\partial x_{j}} n_{i} d V=0
$$

where $\delta c_{v}$ is the test function for the concentration variable and

$$
\int_{V} \delta \mu \mu d V-\int_{V} \delta \mu\left(\frac{\partial \psi}{\partial c_{v}}=\frac{E_{f}}{\Omega_{0}}+\frac{R T}{\Omega_{0}} \log \left(\frac{c_{v}}{1-c_{v}}\right)-\eta_{i j} \sigma_{i j}\right) d V=0
$$

where $\delta \mu$ is the test function for the chemical potential.

\subsubsection{Example}

The model is tested on a square grain with a length of $25 \mu \mathrm{m}$ as shown in Fig.11a. A normaldistribution function is used to define the grain boundary order parameter and is shown in Fig.11b. The grain is loaded in tension on the top face and compression on the right face with a normal traction of $100 \mathrm{MPa}$. A temperature of $1000^{\circ} \mathrm{C}$ is used to derive some of the parameters. The rest are obtained from [17]. Assuming, that initially $(\mathrm{t}=0)$ the free energy is zero, the equilibrium concentration is calculated from Eq.10 as $1.4 \times 10^{-8}$. The equilibrium concentration is maintained on the GB using a Dirichlet boundary condition.

Once the biaxial traction is applied, the top and bottom surfaces acts as vacancy sources, while the right and left faces act as sinks. This is due to the increase and decrease of chemical potential due to the tensile and compressive stresses, respectively, on the GB faces (Fig.12a). Due to the source/sink nature of the GBs, the concentration of vacancies increase or decrease in the representative volume and is evident from the spatial distribution shown in Fig.12b. The net flux of vacancies towards the top/bottom faces and away from the left/right faces is also observed in Fig.13 consistent with the concentration and chemical potential distribution. The equivalent creep strain is shown in Fig.14a. As can be observed, regions near the triple points have larger creep strain and can be due to the multi-directional vacancy fluxes in these regions. The evolution of the volume averaged equivalent creep strain with time is shown in Fig.14b. 


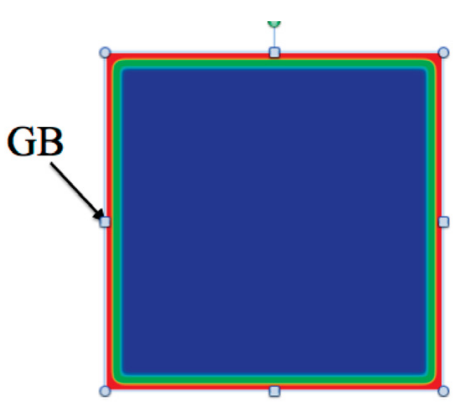

(a)

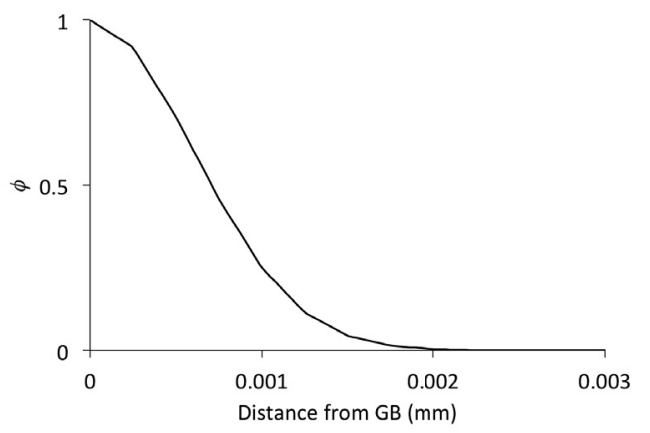

(b)

Figure 11: (a) Geometry of the square domain; (b) Grain boundary order parameter.

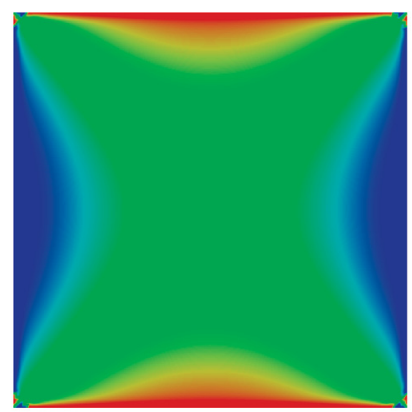

(a)

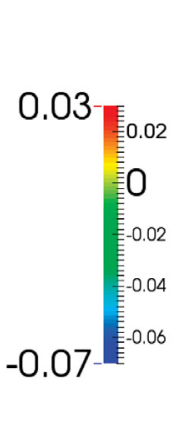

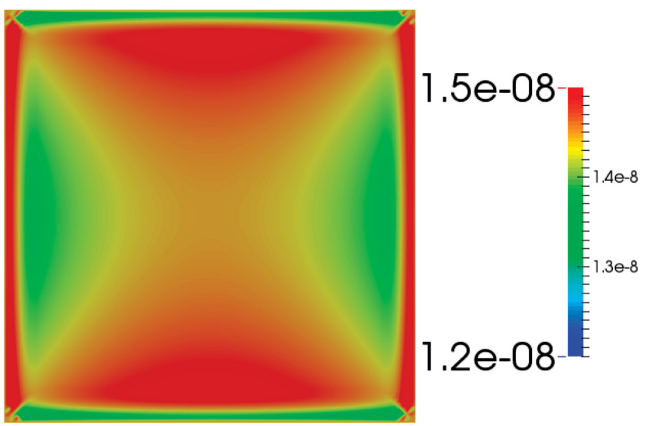

(b)

Figure 12: (a) Chemical potential and (b) concentration distribution at $t=100$ sec.

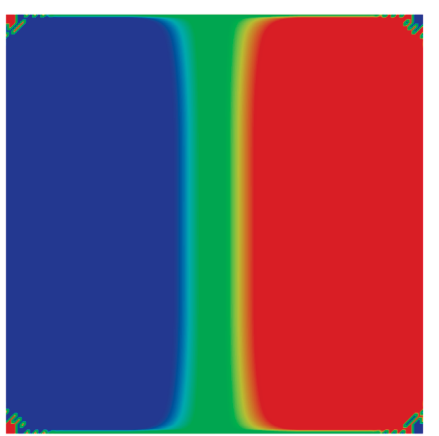

(a)

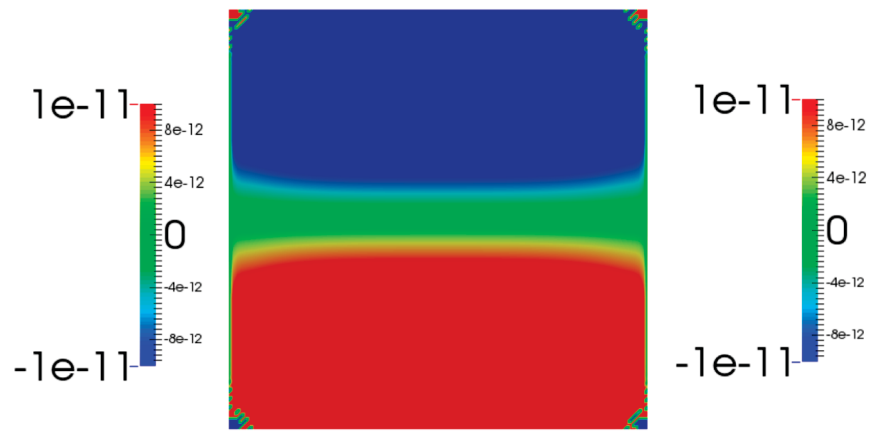

(b)

Figure 13: Vacancy flux along (a) $\mathrm{x}$ and (b) y directions 


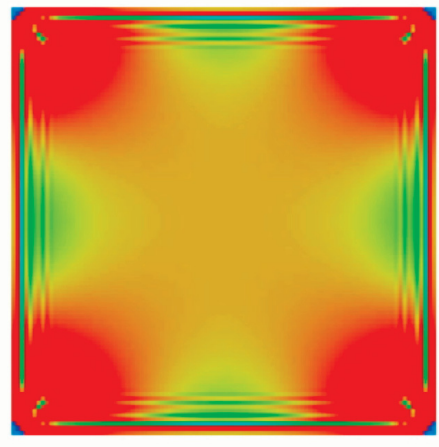

(a)

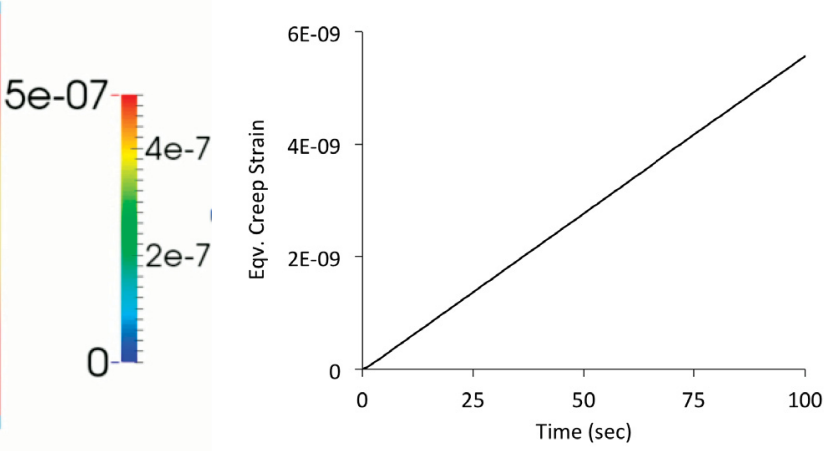

(b)

Figure 14: (a) Spatial distribution of equivalent creep strain; (b) Temporal evolution of volume averaged equivalent creep strain.

\subsection{Comparison between Phase-field and XFEM models for brittle fracture}

The phase-field fracture model developed in FY14/15 is based on a critical energy release rate parameter and principal strains. Though, the phase-field fracture model has been compared with linear elastic fracture mechanics (LEFM) extensively for mode-I problems, a systematic study on the similarities and differences for mixed-mode problems is missing. In this section such a comparison is made with XFEM based fracture model both quantitatively (e.g. force-displacement) and qualitatively (e.g. crack-path).

In the first comparison, a symmetric center crack geometry under mode-I loading is considered. The geometry, boundary conditions and force-displacement is shown in Fig.15. In this comparison, a maximum principal stress criterion is used in X-FEM. As can be observed from the figure, the phase-field fracture model overestimates the fracture strength and is consistent with previous observation.

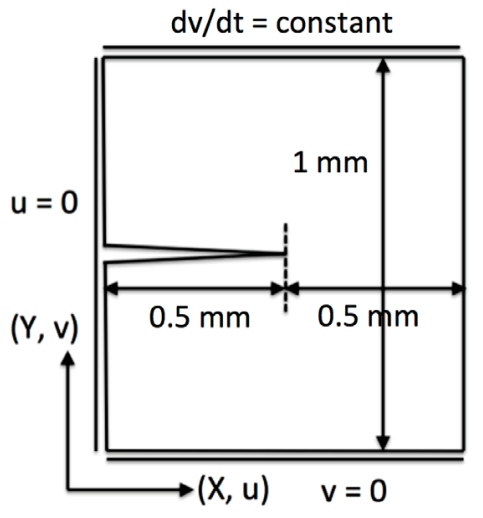

(a)

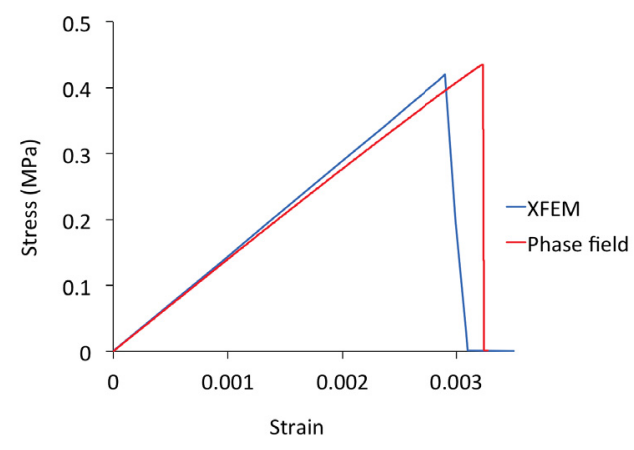

(b)

Figure 15: Symmetric center crack: (a) Geometry; (b) Comparison of stress-strain.

In the second comparison, pre-cracks at different inclinations are considered and the deviation from self-similar crack propagation is measured. In Fig.16, a comparison between experiments, 
phase-field fracture and X-FEM is shown. For X-FEM, both configurational force (METHOD 1) and maximum circumferential stress from interaction integral (METHOD 2) methods have been used. As can be observed from the figure, phase-field fracture and X-FEM provide very similar results for inclination angles between 30 and 90 degrees. However at lower inclinations, the crack propagation directions significantly differs. As can be observed from Fig.16c, the crack path predicted by X-FEM has better agreement than the phase-field fracture model (Fig.16b).

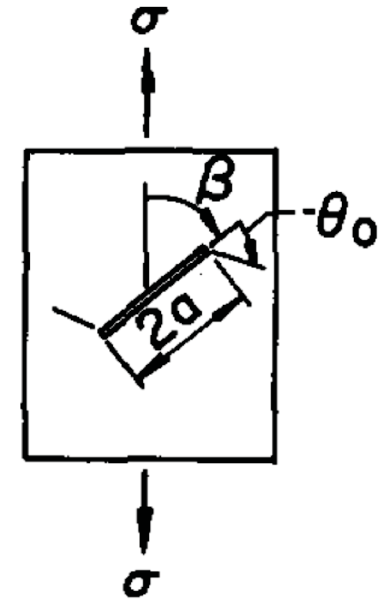

(a)

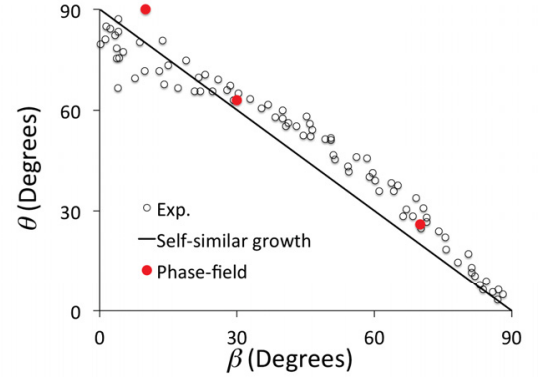

(b)

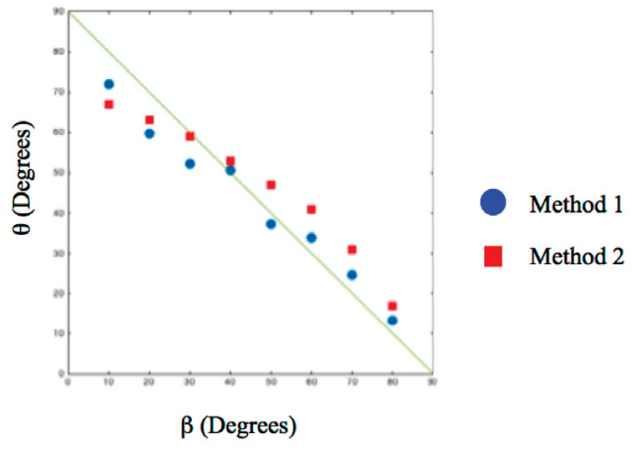

(c)

Figure 16: (a) Geometry of the inclined crack. Deviation from self similarity (b) phase-field fracture and (c) X-FEM. In (c), METHOD 1 and 2 are based on configurational force and maximum circumferential stress from interaction integral.

In the third comparison, the Bittencourt problem is considered that consists of a 3-point bend specimen with non-symmetrical holes and pre-crack. A schematic is shown in Fig.17. In this example, both mode-mixity as well as interaction of the propagating cracks with the holes, determine the final crack path. Two different pre-crack locations are considered and the comparison is shown in Fig.18 and 19. As can be observed from the figures that though the initial crack paths are similar between X-FEM and phase-fields, the paths deviate close to the holes. This deviation can be due to diffuse damage occurring near the holes. Also, the thickening of the damage region in the phase-field model may cause this deviation.

From the comparison it can be concluded that the finite thickness of the phase-field fracture model may result in deviation from experimentally observed crack paths. However, for most of the 


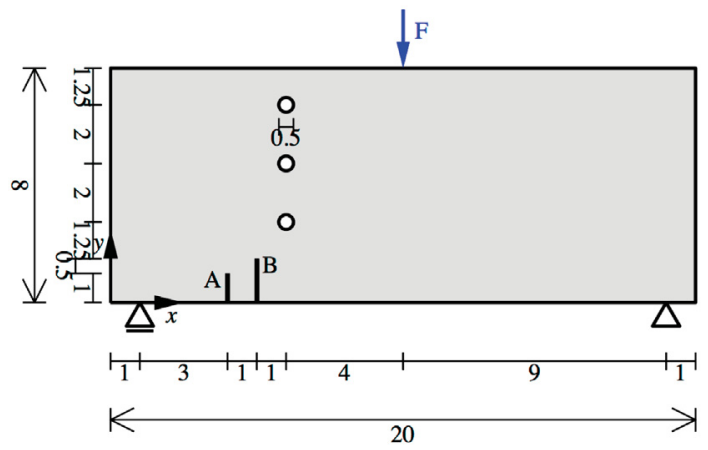

Figure 17: Schematic of the Bittencourt problem

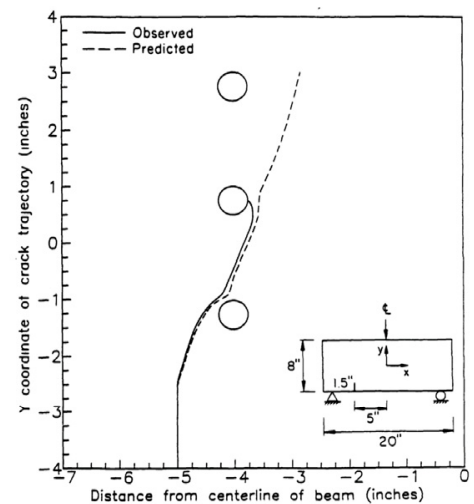

(a)

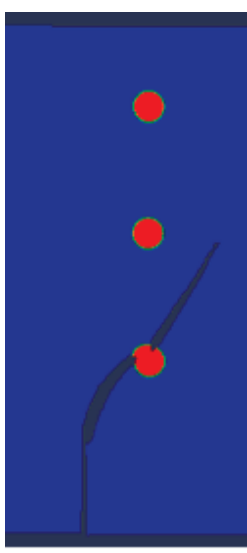

(b)

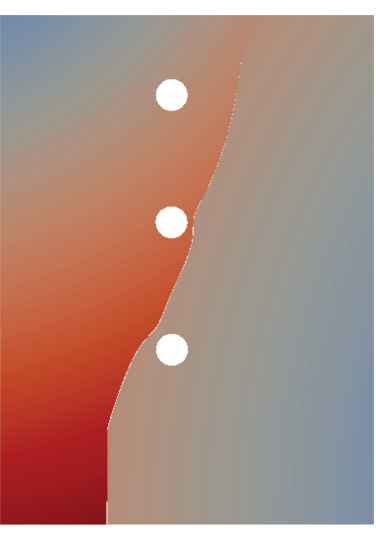

(c)

Figure 18: (a) Experiment, (b) Phase-field fracture and (c) X-FEM

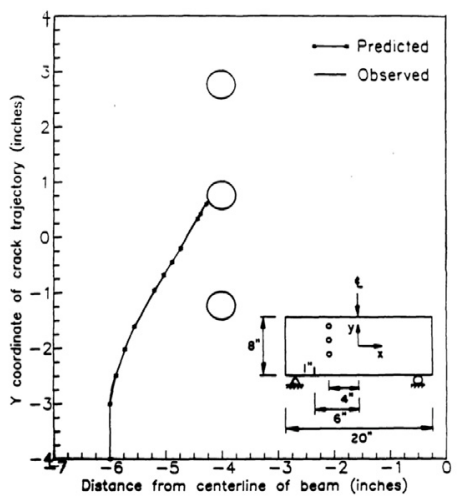

(a)

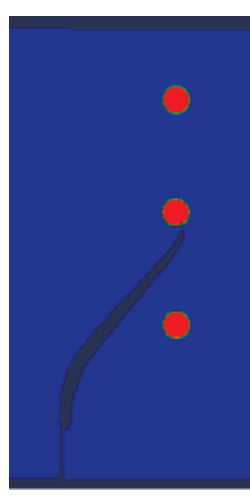

(b)

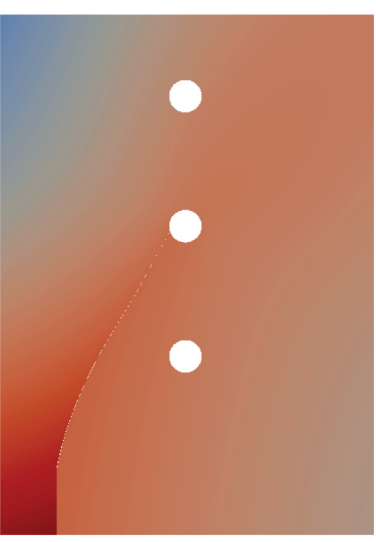

(c)

Figure 19: (a) Experiment, (b) Phase-field fracture and (c) X-FEM 
cases the model provides reasonable results. Though, tracking of crack-path in X-FEM in 3D is a major limitation, it has shown better comparisons in the $2 \mathrm{D}$ mixed mode problems considered here. 


\section{Thermodynamics of solid fission products in $\mathrm{UO}_{2}$ 7.1 Introduction}

In light water nuclear reactors (LWR), energy is generated from the fission of U-235 atoms in $\mathrm{UO}_{2}$ fuel pellets. Predicting the chemical reactions of fission products with fuel and cladding is an important part of fuel performance modeling. Among more than 50 elements, $\mathrm{Zr}, \mathrm{Mo}, \mathrm{Nd}, \mathrm{Ru}$, Xe, $\mathrm{Cs}, \mathrm{Ce}, \mathrm{Sr}, \mathrm{Ba}, \mathrm{La}, \mathrm{Tc}, \mathrm{Pd}, \mathrm{Pr}$ and $\mathrm{Y}$ are the major fission products of U-235 with the highest yields [18]. These fission products can exist in various forms including gas bubbles (Xe), oxide inclusions, oxides dissolved in $\mathrm{UO}_{2}$ matrix, and metallic inclusions. With increasing burnup, the initially single-phase $\mathrm{UO}_{2}$ fuel will evolve into a complex multicomponent and multiphase system.

The objective of the present study is to better understand the thermodynamic equilibrium states of the major solid fission products in $\mathrm{UO}_{2}$. Our initial focus is on the thermodynamics of the binary systems formed between the major solid fission products of U-235. All binary sub-systems of the 13-component Zr-Mo-Nd-Ru-Cs-Ce-Sr-Ba-La-Tc-Pd-Pr-Y system are considered. Results from our literature review and ab initio calculations are reported in this section.

\subsection{Review of binary phase diagrams}

We have reviewed the existing experimental phase diagrams [19] for the binary sub-systems of Zr-Mo-Nd-Ru-Cs-Ce-Sr-Ba-La-Tc-Pd-Pr-Y, and our results are summarized in Figure 20. The experimental phase diagrams are classified into four types:

Type A: The system forms continuous solid solution at high temperature. At low temperatures, miscibility gap or ordered compounds may possibly form.

Type B: Strongly ordered intermetallic compounds exist in the system, which remain stable from low temperature up to melting without disordering. Compounds that are only stable at high temperatures, e.g. the $\sigma$ phase in Fe-Cr, are not considered as being strongly ordered since they will decompose at low temperatures.

Type C: No compound forms in the system. The mutual solubility between the two end-member elements is also negligibly small. In many of these systems, there even exists a miscibility gap in the liquid phase.

Type D: Extensive solid solutions exist in the system at high temperature. Due to different crystal structures of the two end-member elements, continuous solid solution cannot be formed. No strongly ordered compounds exist in the system.

Typical phase diagrams of types A to D are shown in Figure 21. Among the 49 binary systems considered, only one system, Mo-Tc, defies this classification. In Massalski's version of Mo-Tc [19], there exist extremely large mutual solubility between the Mo and Tc (see Figure 22(a)), which indicates ideal mixing behavior. However, there also exists an ordered $\sigma$ phase with composition $\mathrm{Mo}_{0.3} \mathrm{Tc}_{0.7}$ that is stable up to melting, suggesting an ordering-type system. Interestingly, this ordered $\sigma$ phase does not exist in the other version of the Mo-Tc phase diagram (see Figure 22(b)) [20]. This inconsistency may warrant further experimental and theoretical investigations. In our

study, the Mo-Tc system is classified as type D since it is unclear if the $\sigma$ phase will remain stable down to low temperatures. 


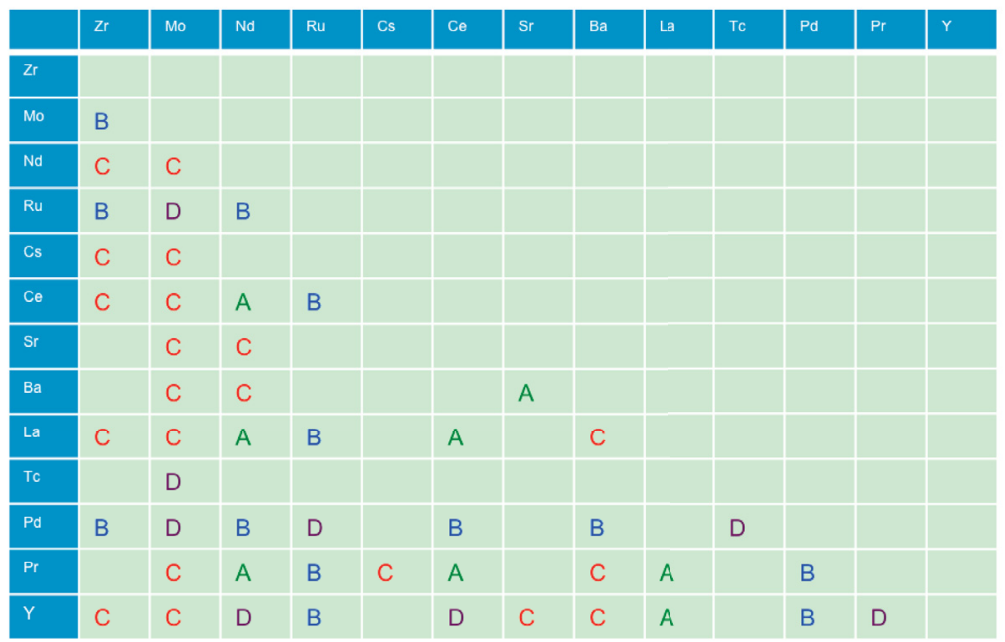

Figure 20: Literature review of the phase diagrams of binary systems between the major solid fission products of U-235.

\subsection{Density Functional Theory calculations of thermodynamic properties}

In this work, ab initio calculations based on density functional theory (DFT), as implemented in the Vienna ab initio simulation package (VASP) code [21], have been performed to obtain the mixing energies of the random solid solution phases at the equiatomic composition in all 78 binary sub-systems of Zr-Mo-Nd-Ru-Cs-Ce-Sr-Ba-La-Tc-Pd-Pr-Y. All calculations are performed within the generalized gradient approximation (GGA) with the Perdew-Burke-Ernzerhof (PBE) exchangecorrelation function [22]. The plane wave cutoff energy is set at $350 \mathrm{eV}$. The $k$-point meshes for Brillouin zone sampling are constructed using the Monkhorst-Pack scheme and the total number of $k$-points times the total number of atoms per unit cell was at least 5000 for all structures. We fully relax the unit cell volumes of all structures with atoms occupying their ideal lattice positions. The relaxation of internal atomic positions is intentionally prohibited to avoid possible issue of mechanical instability.

We calculate the mixing energy of a disordered alloy $\mathrm{A}_{1-x} \mathrm{~B}_{x}$ in crystal structure $\phi$ as:

$$
\Delta H_{\text {mix }}^{\phi}=E\left(A_{1-x} B_{x}\right)-(1-x) E(A)-x E(B)
$$

where $\mathrm{E}(\mathrm{A})$ and $\mathrm{E}(\mathrm{B})$ are the total energies of pure $\mathrm{A}$ and $\mathrm{B}$, both in structure $\phi$, respectively. According to the study of Martins and Zunger [23], the mixing energy can be decomposed into the following two parts:

$$
\Delta H_{\text {mix }}=\Delta H_{\text {strain }}+\Delta H_{\text {chem }}
$$

where the strain energy $\Delta H_{\text {strain }}$ is due to the different sizes of $\mathrm{A}$ and $\mathrm{B}$ atoms. In latticemismatched alloys, this is the dominant term leading to phase separation. The chemical interaction energy $\Delta H_{\text {chem }}$ is due to the formation of new A-B bonds from A-A and B-B bonds, which depends on the difference in Pauling electronegativity between A and B atoms. While the strain energy is always positive and favors phase separation, the chemical energy can be negative and drive the formation of ordered compounds in a system. Presumably, it is the competition between $\Delta H_{\text {strain }}$ and $\Delta H_{\text {chem }}$ that determines the type of a phase diagram. 

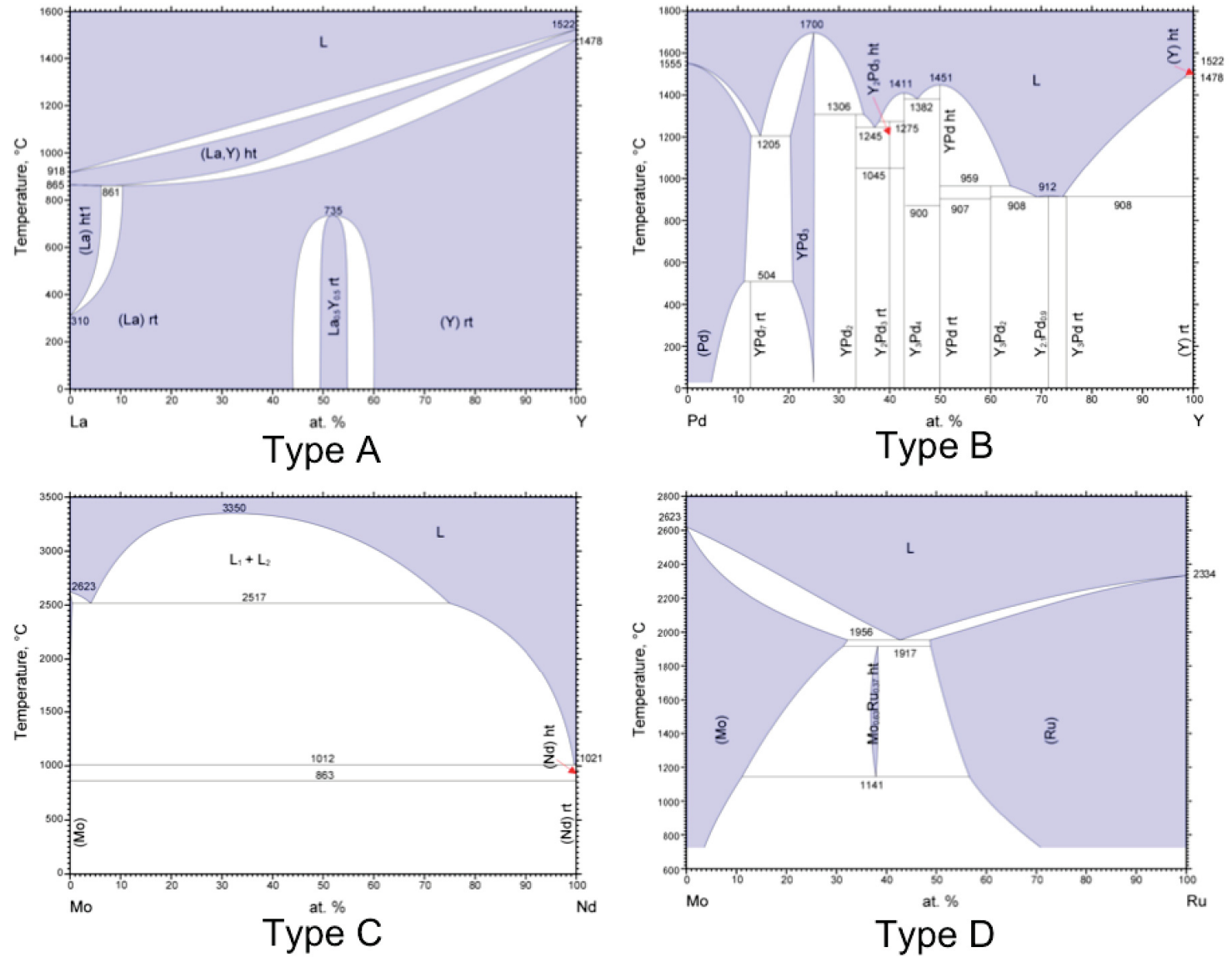

Figure 21: Example phase diagrams of types A to D.

It is worth noting that the electronegativity values of atoms are derived from the dissociation energies of chemical bonds in molecules and are assumed to be transferable to other chemical environments. In a solid, however, the bond strength can vary with the local environment and is usually weakened with increasing coordination. Such bond-order effects are not considered in the original electronegativity concept.

\subsection{Results}

Figure 23 shows the $\Delta H_{\text {chem }}$ vs. $\Delta H_{\text {strain }}$ plot for the 49 binary systems with known experimental phase diagrams. DFT results for the equiatomic bcc solid solution phases are shown. Remarkably, with the exception of Mo-Zr, different phase diagram types are clearly separated into different domains in this two-dimensional plot. In order to form type $\mathrm{A}$ and type $\mathrm{D}$ phase diagrams that are dominated by solid solution phases, both $\Delta H_{\text {strain }}$ and $\left|\Delta H_{\text {chem }}\right|$ need to be small. This is consistent with the well-known Hume-Rothery rules, which state that a binary X-Y system will show large mutual solubility if the $\mathrm{X}$ and $\mathrm{Y}$ atoms have similar atomic radius and electronegativity. Type B phase diagrams will form when the chemical energy is strongly negative. In a X-Y system with $\Delta H_{\text {chem }} \ll 0$, ordered compounds will still form even when there exists large size mismatch between $\mathrm{X}$ and $\mathrm{Y}$ atoms, leading to $\Delta H_{\text {strain }} \gg 0$ in a solid solution. A good example is the Ce-Ru system. The lattice parameters of bcc Ru and bcc Ce are calculated by DFT to be $3.06 \AA$ and $4.21 \AA$, respectively. The size mismatch between $\mathrm{Ce}$ and $\mathrm{Ru}$, calculated as $\Delta a / \bar{a}$, is as large as 

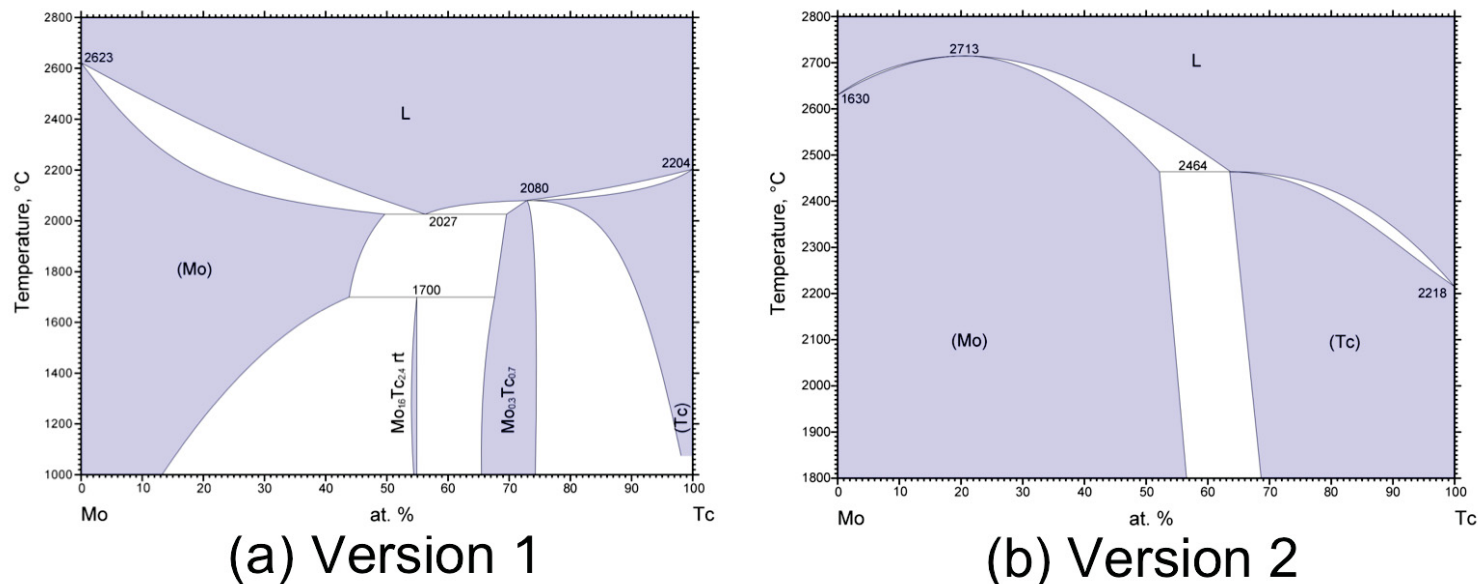

Figure 22: Two versions of the Mo-Tc phase diagram. Version 1 is from REF.Massalski1990 and version 2 is from REF.Haines1980

$32 \%$. Despite this, many ordered compounds including $\mathrm{Ce}_{3} \mathrm{Ru}, \mathrm{Ce}_{4} \mathrm{Ru}_{3}$ and $\mathrm{CeRu}_{2}$ form in this system. This suggests that the strain energy is not a good predictor for the formation of ordered compounds in a system. Finally, type $\mathrm{C}$ phase diagrams will form under two situations. In the first case, the chemical energy itself is strongly positive, and phase separation will occur regardless of size mismatch. In the second case, a large positive strain energy dominates the thermodynamics of the system, and the magnitude of the chemical energy contribution is much smaller in comparison.

While Mo-Zr is classified as a type B system due to the formation of an ordered $\mathrm{ZrMo}_{2} \mathrm{Laves}$ phase that remains stable from low temperature up to melting, it actually falls within the type $\mathrm{C}$ domain where phase separation occurs (see Figure 23). Interestingly, according to two recent thermodynamic modeling of the Mo-Zr system [24, 25], the bcc phase in the Mo-Zr system indeed has a tendency to phase separate due to the existence of a metastable miscibility gap at high temperatures. Mo-Zr is thus an unusual system exhibiting both ordering (type B) and phase separation (type $\mathrm{C}$ ) tendencies.

Finally, the present DFT results can be used to provide insights into the chemistry of the metallic fission-product precipitates, also called white inclusions, in irradiated uranium oxide fuels. Experimentally, it was observed that the white inclusion is a Mo-Ru-Tc-Pd-Rh solid solution with hcp crystal structure [18]. Since Rh is not considered in this work, we focus on the four-component Mo-Ru-Tc-Pd system instead. Figure 24 shows the $\Delta H_{\text {chem }}$ vs. $\Delta H_{\text {strain }}$ plot for the 6 binary subsystems of Mo-Ru-Tc-Pd. For all of them, the calculated $\Delta H_{\text {chem }}$ and $\Delta H_{\text {strain }}$ values are indeed rather small, which favors the formation of solid solution phases (type A or type D behavior). Our DFT calculations thus provide direct support of the experimental observation. While the RuTc phase diagram does not exist in the literature, we predict it to be a type A system in view of its very small strain $(0.002 \mathrm{eV} /$ atom $)$ and chemical $(0.007 \mathrm{eV} /$ atom $)$ energies. Such a conclusion is in agreement with an experimental study using X-ray diffraction [26] indicating the formation of continuous solid solution in this system at $973 \mathrm{~K}$.

\subsection{Summary}

In this work, we have performed a literature review of the binary systems formed between the major solid fission products of U-235. DFT calculations have been performed to obtain the thermodynamic properties of all 78 binary sub-systems of the 13-component Zr-Mo-Nd-Ru-Cs-Ce-Sr-Ba-La-Tc-Pd- 


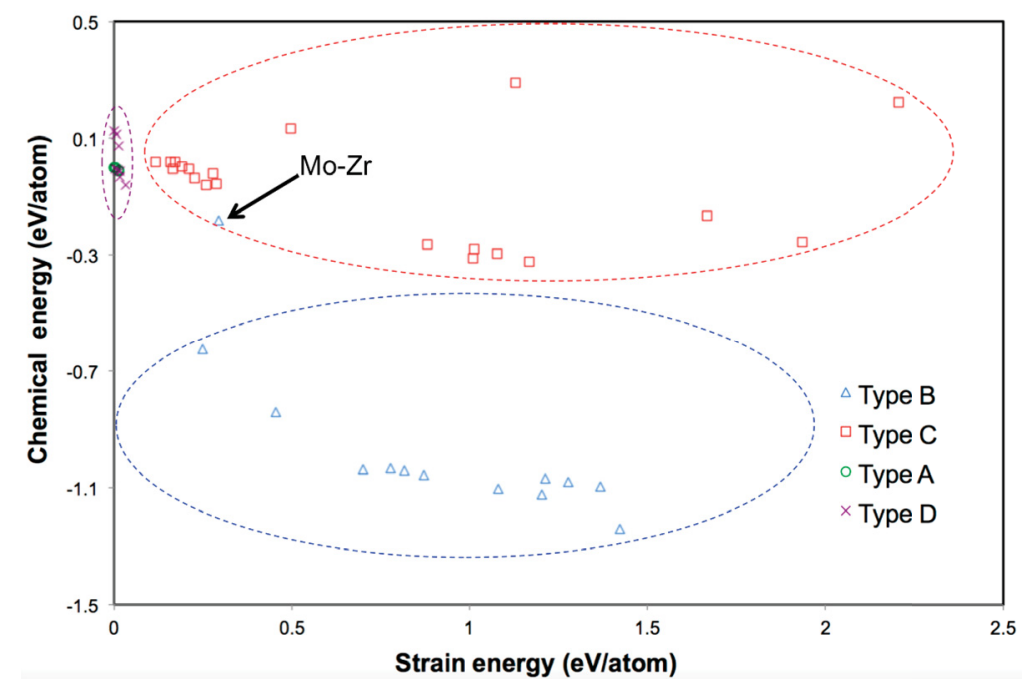

Figure 23: $\Delta H_{\text {chem }}$ vs. $\Delta H_{\text {strain }}$ plot for the binary sub-systems of Zr-Mo-Nd-Ru-Cs-Ce-Sr-BaLa-Tc-Pd-Pr-Y.

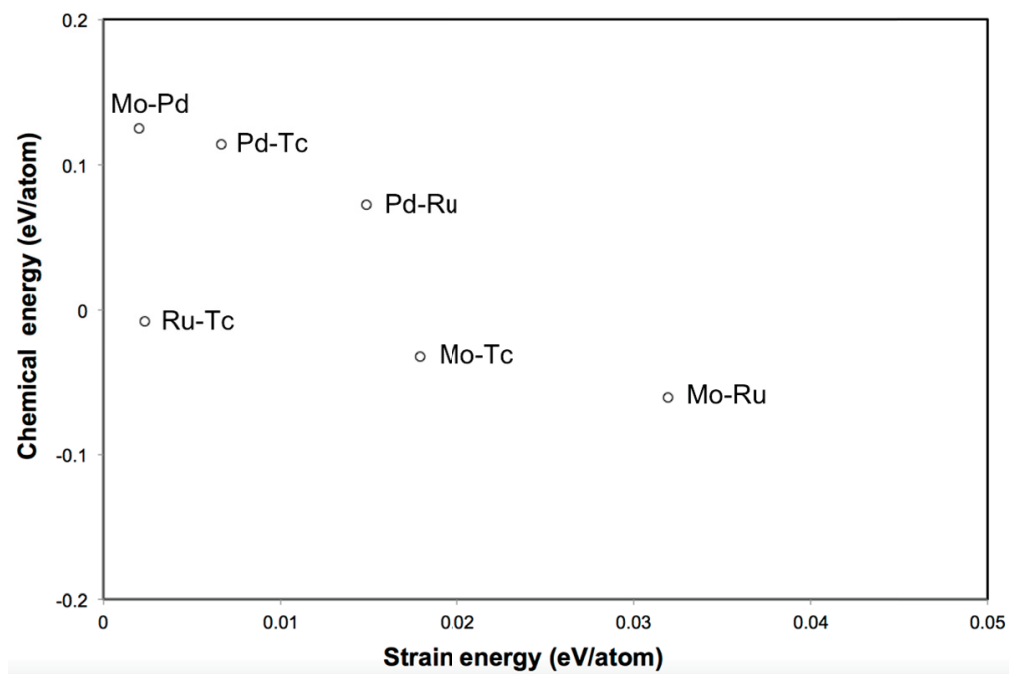

Figure 24: $\Delta H_{\text {chem }}$ vs. $\Delta H_{\text {strain }}$ plot for the binary sub-systems of Mo-Ru-Tc-Pd.

Pr-Y system. Such results may be used as inputs to drive phase field simulations in MARMOT. By decomposing the mixing energy into strain energy and chemical energy contributions, our DFT calculations can predict with a high successful rate if a system exhibits ordering (type B), phase separation (type C) or ideal mixing (type A and type D) behavior. Our DFT results also give direct support of the experimentally observed composition of the white inclusions in irradiated $\mathrm{UO}_{2}$.

In our future study, reactions between the solid fission products with $\mathrm{UO}_{2}$ will be considered. Specifically, we will consider the solubility of solid fission products in $\mathrm{UO}_{2}$ as well as the possible oxides of the fission products, which will result in more accurate prediction of the thermodynamic equilibrium states of the major solid fission products in $\mathrm{UO}_{2}$. 


\section{Microstructure-based thermal conductivity \\ 8.1 Introduction}

The fuel thermal conductivity decreases throughout the lifetime of the fuel due to evolution of the microstructure. Traditional fuel performance tools use empirical models for the thermal conductivity that are correlated to temperature and burn-up. The NEAMS fuel product line has been developing a mechanistic model of the fuel thermal conductivity as part of a larger effort to develop improved materials models that are based on microstructure rather than burn-up. Significant progress has been made on the thermal conductivity model, accounting for the impact of various types of fission gas $[27,28]$ and coupling it to the fission gas release model in BISON. However, the model is not yet complete. It also needs to account for the impact of point defects and other fission products in the fuel.

\subsection{Work during FY 2016}

\subsubsection{Effect of point defects on thermal conductivity}

This year we have begun to include the impact of point defects within the fuel on the thermal conductivity. Due to irradiation damage, uranium (U) and oxygen (O) atoms are displaced from their lattice sites, creating vacancies on both the $\mathrm{U}$ and $\mathrm{O}$ sublattices. These displaced atoms create interstitial defects on the two sublattices. These point defects (both vacancies and interstitials) increase phonon scattering in the fuel, decreasing the fuel thermal conductivity. Thus, it is critical to account for this decrease in our mechanistic model of the fuel thermal conductivity.

The mechanistic fuel thermal conductivity model takes the form

$$
k=\kappa_{I G}\left(p_{i g}\right) \kappa_{G B}\left(f_{c}\right)\left(k_{L}\left(t, c_{f g}, c_{P D}\right)+k_{A P}(t)\right)
$$

where $t=\frac{T(K)}{1000}, \kappa_{I G}$ accounts for intragranular gas bubbles, $\kappa_{G B}$ accounts for the impact of grain boundaries and grain boundary gas bubbles, $k_{L}$ accounts for the phonon lattice contribution and is a function of temperature and fission gas and point defect concentration $c_{P D}$ and $c_{f g}$, and $k_{A P}$ accounts for the small polaron contribution. This model directly couples to the fission gas release model, which calculates the dispersed fission gas concentration $c_{f g}$, the intragranular bubble density $p_{i g}$, and the percentage of grain boundaries covered by fission gas bubbles $f_{c}$.

This year, we have focused on the phonon lattice contribution

$$
k_{L}=\left(A+B T+C T^{2}+K_{X e} c_{X e}+K_{V_{U}} c_{V_{U}}+K_{I_{U}} c_{I_{U}}+K_{V_{O}} c_{V_{O}}+K_{I_{O}} c_{I_{O}}\right)^{-1} .
$$

The values for $A, B$, and $C$ are taken from experiments. The other terms account for additional phone scattering due to different defect concentrations, the concentrations of xenon $c_{X e}$, $\mathrm{U}$ vacancies $c_{V_{U}}$, U interstitials $c_{I_{U}}$, $\mathrm{O}$ vacancies $c_{V_{O}}$, and $\mathrm{O}$ interstitials $c_{I_{O}}$. The corresponding coefficients must be obtained using atomistic simulation, as was done for $K_{X e}$ in FY 2015.

This year, the values for the coefficients $K_{V_{U}}, K_{I_{U}}, K_{V_{O}}$, and $K_{I_{O}}$ were obtained by our collaborators at LANL. With these coefficients we can now fully account for the impact of the point defects on the thermal conductivity, if we know their local concentrations throughout the fuel. However, these concentrations are not current being calculated by BISON.

We have begun to develop the necessary models to compute the local point defect concentrations throughout the life time of the fuel pellet. The O-lattice defects migrate at much faster rates than the U-lattice defects, necessitating different treatment. The U-lattice defects concentrations will be 
predicted locally at every material point throughout the BISON mesh using a rate theory approach. The rate theory model will account for defect generation, recombination, and various types of sinks including fission gas bubbles and dislocation loops. Initial rate theory calculations indicate that the $U$ defect concentrations will quickly reach steady state values that are very small $\left(<1 \times 10^{-6}\right)$ and therefore will likely not significantly impact the thermal conductivity.

The O-lattice defects contribute directly to the stoichiometry of the fuel, which can significantly vary radially across the fuel pellet. The fuel stoichiometry will be modeled at the pellet scale by adding a diffusion equation to the set of equations being solved by BISON. This diffusion equation will account for the impact of concentration and temperature gradients on the $\mathrm{O}$ transport. The values for $c_{V_{O}}$, and $c_{I_{O}}$ will be determined from the stoichiometry. Their values are likely to be much larger than the concentrations of the $U$ defects and could have a significant impact on the thermal conductivity.

\subsubsection{Validation on the effect of grain boundaries}

Thermal conductivity of $\mathrm{UO}_{2}$ is affected by stoichiometry (O/M ratio) and many microstructure features including grain size, porosity and lattice defects. To connect the microstructure characterization and thermal measurements with modeling, mesoscale simulations using the MARMOT code were carried out for better understanding of the contribution from each microstructure feature and from chemistry. The results were compared by that measured by the laser flash method or the physical property measurement system (PPMS) at INL. Here, MARMOT simulations were performed to investigate the effect of grain size in the temperature range of $300 \mathrm{~K}$ to $1500 \mathrm{~K}$. Three grain sizes, $7.2 \mu \mathrm{m}, 1.8 \mu \mathrm{m}$ and $0.125 \mu \mathrm{m}$, were considered, in accordance to the experimental samples. All simulations were performed using a 2-D square domain (Fig.25). The size of the domain varied by the grain size used. The cells for $7.2 \mu \mathrm{m}$ and $1.8 \mu \mathrm{m}$ grain sizes contained 32 grains, and the one for $0.125 \mu \mathrm{m}$ contained 64 grains. To improve computation efficiency, mesh adaptivity capability in MOOSE was activated to give a good resolution at grain boundary regions (Fig.25). After being set up, the simulation cells were relaxed at $1500 \mathrm{~K}$ to quickly reach the equilibrium grain boundary width. After the microstructures were relaxed, the thermal conductivity was calculated using the Fourier's law at desired temperatures by setting up a temperature gradient as shown in Fig.25. In MARMOT calculations for overall thermal conductivity, each microstructural feature is assigned with a different conductivity. The thermal conductivity of $\mathrm{UO}_{2}$ matrix, $K_{0}$, is described using the Fink model [29]. The grain boundary conductivity was set to be proportional to $K_{0}$ with a coefficient of 0.578 which gives a grain boundary thermal conductivity of $5.0 \mathrm{~W} / \mathrm{mK}$ at $300 \mathrm{~K}$ [30]. We note that a temperature dependent conductivity or thermal resistance model for grain boundaries in $\mathrm{UO}_{2}$ is yet to be developed.

The MARMOT simulations were carried out with fully dense $\mathrm{UO}_{2}$. As shown by the results in Fig.25, thermal conductivity of polycrystalline $\mathrm{UO}_{2}$ decreases with decreasing grain size. This is because grain boundaries have a lower conductivity than that of bulk $\mathrm{UO}_{2}$, and the volumetric density of boundaries increases as grain sizes decreases [30]. However, such a trend was not followed in the experimental measurements, due to the fact that the experimental samples were porous and non-stoichiometric, i.e., with $\mathrm{O} / \mathrm{M}$ ratios other than 2.0. From both the PPMS and laser flash measurements, above room temperature the $1.8 \mu \mathrm{m}$ sample has a higher conductivity than the $7.2 \mu \mathrm{m}$ one. To better understand the experimental results, the modeling results were adjusted to account for the effects of porosity and off stoichiometry. Because the experimental samples were with similar densities, the corrections for porosity did not change the trend established by grain size. In contrast, the three samples vary a lot in terms of O/M ratio. Following Hobson et al. [31], the MARMOT results were fitted with respect to temperature $\mathrm{T}$ using $\mathrm{K}=1 /(\mathrm{A}+\mathrm{CT})+\mathrm{BT}^{3}$, with 

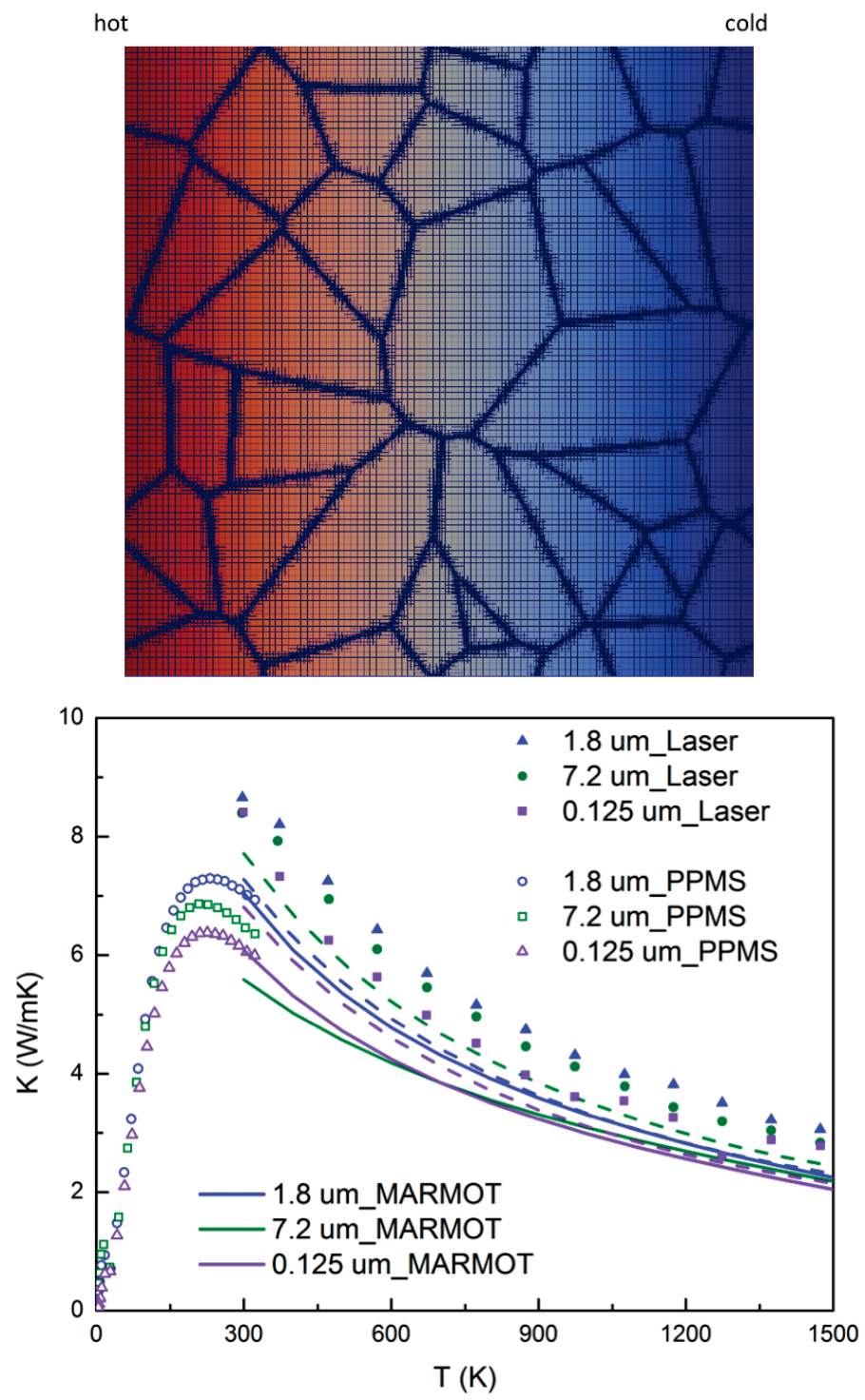

Figure 25: (a) Schematic of a 2D simulation cell for polycrystalline $\mathrm{UO}_{2}$. The grain boundaries are decorated by refined meshes. A temperature gradient is set up from left (hot) to right (cold). (b) Thermal conductivities of $\mathrm{UO}_{2}$ samples with different grain sizes as function of temperature. The dash lines are MARMOT results without corrections for stoichiometry and porosity. The solids lines are after corrections.

$\mathrm{A}, \mathrm{B}, \mathrm{C}$ being fitting parameters. Both $\mathrm{A}$ and $\mathrm{C}$ are $\mathrm{O} / \mathrm{M}$ ratio dependent, with extra contribution from off-stoichiometry. For $\mathrm{UO}_{2-\mathrm{x}}$, extra resistance can be estimated using $\mathrm{A}=\mathrm{A}_{0}+2.85^{*} \mathrm{x}$, and $\mathrm{C}=\mathrm{C}_{0}-7.15 \mathrm{E}-4^{*} \mathrm{x}$, following Popov et al. [32]. While making these corrections, $\mathrm{O} / \mathrm{M}$ ratios were taken from the measurements, which were done before the samples were shipped from the fabricator at Rensselaer Polytechnic Institute (RPI) to INL. After the corrections, the $7.2 \mu \mathrm{m}$ sample was of lower conductivity than the $1.8 \mu \mathrm{m}$ due to its lower $\mathrm{O} / \mathrm{M}$ ratio, i.e., larger $\mathrm{x}$. Therefore the trend observed in MARMOT simulations agreed with that from experiments. The MARMOT results matched those from the PPMS measurements well except for the $7.2 \mu \mathrm{m}$ sample. However, some discrepancies between modeling and experiments, or between different experimental measurements, 
were still noticed regarding the absolute values. Possible reasons include uncertainties in different measuring methods, and the change in fuel microstructure and chemistry during experimental processing. It was suspected that the samples might have been further oxidized during delivery, i.e., the $\mathrm{O} / \mathrm{M}$ ratio could be higher than those measured at RPI. The results shown here demonstrated the capabilities developed for microstructure level characterization, and the importance of doing that for predicting fuel properties and thus fuel qualification. A close tie between modeling and experiments has also been established considering effects of microstructure features such as grain size. The comparison between modeling and experiments also calls for the need of full characterization of the evolving fuel microstructure and chemistry in order to accurately estimate fuel properties such as thermal conductivity.

\subsection{Summary}

The current fuel thermal conductivity model that has been developed as part of the NEAMS fuels product line considers the impact of fission gas of grain boundaries [27, 28]. However, the complete model must also account for other defects. In FY 2016, INL has begun to implement the impact of point defects on the uranium and oxygen sublattices. An attempt to validate the contribution of grain boundaries suggests that detailed characterizations of fuel chemistry and all microstructure features such as porosity and stoichiometry are required for full validation.

\subsection{Future work}

While the addition of the impact of point defects in the fuel thermal conductivity model is complete, models defining the evolution of the defect concentrations will continue to be developed in the next fiscal year. Once the models are completed and implemented in BISON, the BISON assessment cases will be run to evaluate their impact on the prediction of the fuel performance.

Once the point defect model is fully implemented, the final pieces of the fuel thermal conductivity model will be the consideration of other fission products. These include metal and oxide fission product precipitates and dissolved fission products. This development will likely occur in FY 2017

and 2018. A comprehensive validation of the thermal conductivity model will also be carried out in the future. 


\section{Center hole formation in fast reactor fuels \\ 9.1 Introduction}

Nuclear reactors operate at high temperatures and the thermal gradients that develop within the fuel can drive unique microstructural changes [33]. In particular, fast reactor oxide fuel exhibits a unique fuel restructuring evolution that occurs early in its lifetime once temperature gradients exceeding $1000 \mathrm{~K} / \mathrm{cm}$ become established. The initial porosity within the as-fabricated fuel (5 $10 \%$ after compaction sintering) undergoes a radially inward migration due to the steep temperature gradients that leads to the formation of a 'central void' [34]. Experimental observations moreover have documented that a migrating pore's shape can transform into either a lenticular morphology or a prolate (bullet-shaped) morphology depending on the radial location of the pore [35], see Fig.26. This substantial redistribution of porosity alters the grain structure whereby, in the inner one-third of the fuel pellet, the initial equiaxed grain distribution is replaced with larger columnar grains that are inwardly oriented towards the fuel center [34].

The physical origins of thermal gradient pore migration have been considered for decades. Two basic mechanisms have emerged to explain the phenomenon: $(i)$ solid-state thermodiffusion (or Soret-effect diffusion [36]) either at the pore surface or within the solid, and (ii) vapor transport of the solid material across the inside of the pore. In the former mechanism, a temperature gradient acts as a secondary driving force for solid-state diffusion, in addition to the conventional concentration gradient driving force. On the other hand, for vapor transport, the driving force is the variation of the vapor pressure of the solid material with temperature inside the pore. This mechanism requires the evaporation of fuel material on the hot side of the pore and condensation of material on the cold side of the pore. The models of Nichols [37, 38, 39], and of Sens [35] are the most often cited descriptions of the vapor transport mechanism (they are, in fact, quite similar theoretical treatments [40]).

According to theoretical derivations, each mechanism dominates the migration velocity at different regimes of pore size [41]. For example, the pore velocity due to the thermodiffusion mechanism is inversely proportional to the pore diameter, and therefore conceivably dominates the migration of nanoscale cavities and gas-filled bubbles. Indeed, atomistic simulations have captured thermal gradient pore migration of very small cavities ( $2 \mathrm{~nm}$ ) and convincingly demonstrated temperaturegradient induced surface diffusion activity [42]. Conversely, according to theory, the pore velocity due to vapor transport is independent of pore diameter, and therefore it is likely the dominant mechanism for the migration of larger pores ( $>$ several microns). Simulating the migration of larger pores is not possible with atomistic numerical methods, and it is still exceedingly difficult to experimentally observe such pore migration with in-situ techniques (although experimental studies [43] have reproduced lenticular pore migration in a laboratory setting, with measurements that agree well with Sens' vapor transport model [35]).

Rather, mesoscopic simulation methods can provide important insight for this topic. Both phase-field [44, 45, 46, 47] and Monte Carlo methods have been developed to simulate pore migration in a temperature gradient. Each of these works incorporated a thermodiffusion model to drive pore migration, despite the fact that theoretical descriptions predict the vapor transport mechanism to be dominant. In these models, thermodiffusion is implemented either with a temperaturedependent free energy function [44, 45] or with a flux term containing the temperature gradient [46]. Indeed, the simulations produced void migration in the high-temperature direction; however, pore shapes remained unchanged during migration, which is counter to experimental observations that migrating pores transform into highly non-equilibrium geometries (e.g. lenticular or prolate shapes). Computer simulations have yet to capture this drastic change in pore shape during migration. 


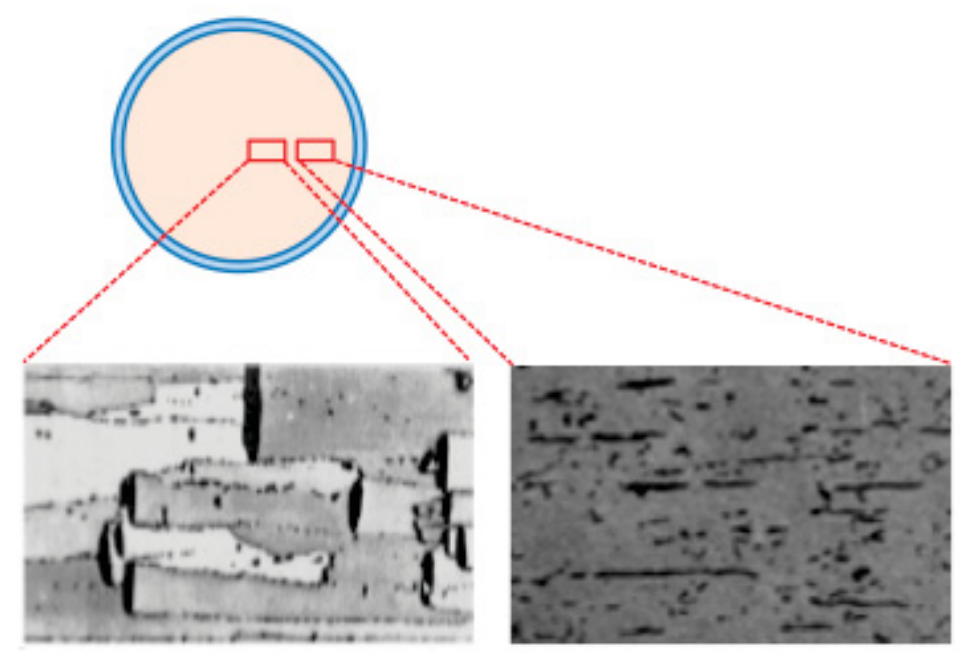

Figure 26: Microscopy images of both lenticular pores and prolate (bullet) shaped pores during thermal-gradient-driven migration in nuclear fuel. Generally, observations show that lenticular pores develop in the inner regions of the pellet and prolate pores develop at the circumference of the columnar grain region, in the middle or outer sections of the pellet.

Moreover, a mesoscale simulation model has yet to be developed that describes pore migration due to vapor transport.

Here, we have developed a phase-field model that incorporates the vapor transport mechanism to simulate thermal gradient pore migration in oxide fuel. In contrast to previous mesoscale simulation studies, our model captures both pore migration towards the high temperature region as well as a concurrent change in the pore shape from an isotropic morphology to either a lenticular morphology or a prolate (bullet-shaped) morphology, consistent with experimental observations. The vapor transport mechanism is integrated into the model by adding an advection term to the Cahn-Hilliard equation that describes the transport of $\mathrm{UO}_{2}$ molecules across the interior of the pore. We implement a vapor transport velocity for $\mathrm{UO}_{2}$ as developed by Sens [35] that enters the advection term that drives pore migration. The simulations reveal that the pore shape changes to a lenticular morphology when the transport velocity has a negative slope in the radial direction, which occurs in the innermost regions of the fuel pellet. Alternatively, the pore shape changes to a prolate morphology when the transport velocity has a positive slope in the radial direction, which occurs in the middle regions of the fuel pellet. Hence, we show that the key to pore shape change is the nature of the slope of the vapor transport velocity in the radial direction. Both of these pore shapes, and their relative positions in the radial direction, agree with experiments [35]. The predictions made by this phase-field model lend further support to the early models that predict the vapor transport mechanism dominates pore migration evolution during fuel restructuring.

\subsection{Simulation methodology}

Before describing the phase-field model, we start by discussing the previous theoretical descriptions of vapor-transport pore migration. The derivation of Sens [35] begins with the assumption that vapor pressure within the as-fabricated pores is independent of surface tension and rather only a function of the local temperature (this is primarily justified by the fact that migrating pores are far from spherically shaped, so an equilibrium pressure $2 \gamma / r$ cannot be applied). By solving a 
diffusion equation describing vapor transport due to gradients in temperature and pressure, and by utilizing an empirical description of $\mathrm{UO}_{2}$ vapor pressure, Sens derived an expression for the transport velocity of gaseous $\mathrm{UO}_{2}$ as a function of temperature and temperature gradient (see Eq. 25 in [35]:

$$
\mathbf{v}=A\left(B+C T+D T^{2}+E T^{3}\right) T^{-5 / 2} \Delta \mathrm{H} p_{o} \exp \left(-\frac{\Delta H}{\mathrm{RT}}\right)\left(\frac{\mathrm{dT}}{\mathrm{dr}}\right)_{\text {matrix }},
$$

where $T$ is temperature, $\Delta H$ is the enthalpy of vaporization of $\mathrm{UO}_{2}, p_{o}$ is a pressure prefactor, and $A-E$ are empirical constants. Using values for $A-E, \Delta H$, and $p_{o}$ prescribed by Sens, the transport velocity can be plotted versus the fuel radial distance as shown in Fig. 27. Here, the temperature is assumed to conform to a parabolic profile with a centerline value of $2800 \mathrm{~K}$ and an outer value of $900 \mathrm{~K}$ (values that are appropriate for fast reactors rather than LWR reactors). Due to the assumption that vapor pressure is independent of surface tension, this velocity profile of $\mathrm{UO}_{2}$ vapor also represents the pore velocity as a function of radial position. Note that the velocity is negative corresponding to a migration direction from right to left on the x-axis (i.e., towards the fuel centerline given that the positive x-direction points from the fuel center to fuel periphery).

Conventional phase-field models describe mass transport and interface migration by solid-state diffusion processes as specified by the Cahn-Hilliard equation [48]:

$$
\frac{\partial c}{\partial t}=\nabla \bullet(M \nabla \mu)
$$

where $c$ is a conserved variable often representing the concentration of a chemical species, $M$ is the mobility of that species, and $\mu$ is the chemical potential of that species defined by a GinzburgLandau energy functional:

$$
\mu=\frac{\delta \mathrm{F}}{\delta \mathrm{c}}=\int\left(f(c)+\kappa|\nabla c|^{2}\right) \mathrm{dV}
$$

where $f(c)$ is a free energy density associated with that chemical species.

On the other hand, mass transport of a dissolved species within a convecting gas or liquid is known as advection and is represented as

$$
\frac{\partial c}{\partial t}=-\nabla \bullet(\mathbf{v} c)
$$

where $c$ is again a conserved variable and $\mathbf{v}$ is the velocity field of a fluid that is transporting the dissolved species.

Therefore, to simulate the vapor transport mechanism governing pore migration, we propose the following Cahn-Hilliard diffusion-advection equation:

$$
\frac{\partial c}{\partial t}=\nabla \bullet(M \nabla \mu)-\nabla \bullet(\mathbf{v} c) .
$$

Here, the velocity field $\mathbf{v}$ can be assigned the expression derived by Sens for the vapor transport velocity, as shown in Eq. 21. We assume the variable $c$ represents a vacancy concentration in the $\mathrm{UO}_{2}$ material and also that a pore is a cluster of vacancies such that within a pore $c \approx 1[49]$. We take advantage of the inherent ability of the Cahn-Hilliard model to evolve interfacial migration within multi-phase microstructures by utilizing a simple double-well free energy density expression $f(c)=c^{2}(1-c)^{2}$ with local minima at $c=0$ and $c=1$. We acknowledge that more physics-based expressions may be used for $f(c)$, for example a regular solution model $[49,50]$. The emphasis of this paper however is to explore the combined mechanisms of diffusion and advection with a 
directional driving force that leads to the one-dimensional migration of a secondary phase, in this case a void.

We solved Eq. 25 using the semi-implicit spectral method [51] for the diffusion term (the first term on the right-hand side) and a central finite difference approximation for the advection term. Two-dimensional simulations were executed in which a uniform rectilinear grid with $512 \times 128$ nodes (or in some cases $1024 \times 128$ nodes) was used for discretization. In order to minimize the impact of round-off errors, reduced units for length and time were chosen to execute the simulations, in which the grid spacing parameters $\Delta \mathrm{x}=\Delta \mathrm{y}=1.0$. To revert to physical lengths, these units correspond to $\Delta \mathrm{x}=\Delta \mathrm{y}=500 \mathrm{~nm}$, therefore the simulation boxes represent $256 \times 64 \mu \mathrm{m}$ (or for the larger domains $512 \times 64 \mu \mathrm{m}$ ) regions within the fuel. The $\mathrm{x}$-direction corresponds to the radial direction, and this is the direction along which the velocity field gradient is present. We used a time step value of $\Delta t=0.1$ reduced units; the reduced time unit corresponding to 40 seconds.

In the simulations, the imposed transport velocity field is also represented in reduced units. Two different velocity field profiles were implemented for two different sets of simulations:

$$
\begin{gathered}
v_{x}=-0.012 x \\
v_{x}=0.005 x-3.1
\end{gathered}
$$

and $v_{y}=0$ throughout the domains. These $\mathrm{x}$-direction velocity fields are linear functions that have negative values throughout the domain (hence pores always travel in the negative radial direction), but one has a negative slope and the other has a positive slope. These two velocity profiles represent the two different shaded regions in Fig. 27. When these two linear velocity profiles are re-scaled into physical units of centimeters and seconds, they can be plotted and compared with Eq. 21 - Sens' model - as is shown in Fig.28. Equation (6) overlaps well in the innermost region of the fuel radius, hence simulations with this velocity profile correspond with radial distances from 0.0 to $0.0256 \mathrm{~cm}$ in the radial direction. On the other hand, simulations using Eq. 27 correspond with the window from 0.185 to $0.2106 \mathrm{~cm}$ in the radial direction (note that in Fig. 28, we shifted Eq. 27 to the right by a distance of $0.2 \mathrm{~cm}$ while keeping the slope constant). All simulations are run for 3000 time steps.

\subsection{Results and Discussion}

The simulations presented here characterize the migration of a single pore with an initially circular shape. Figure 29 depicts successive snapshots of a pore as it migrates across the domain in the x-direction. This particular simulation was run with a $1024 \times 128$ grid (i.e., the larger grid) with the velocity profile specified by Eq. 26. The pore has an initial diameter of $30 \Delta x$, or in physical units $15 \mu \mathrm{m}$, and its center is initially located at $\mathrm{x}=900 \Delta \mathrm{x}$. When using the velocity profile of Eq. 26, the simulation domain corresponds to a region adjacent to the centerline of the fuel, in which the left side of the domain is the fuel centerline and the right side of the domain is 512 $\mu \mathrm{m}$ into the radial direction. Due to the fact that the $\mathrm{x}$-component of the velocity field is negative throughout the domain, the pore migrates in the negative $\mathrm{x}$-direction (in a similar spirit to the axial definitions of Fig. 27). More notably, the pore shape transforms from a circle to a lenticular shape during the migration process. This morphology change is a direct result of the slope of the transport velocity in the $\mathrm{x}$-direction. The velocity profile is negative and also has a negative slope throughout the domain, therefore the leading surface of the pore experiences a lower (or, less negative) velocity than the trailing surface. As a result, the width of the pore in the $\mathrm{x}$-direction reduces due to the fact that the trailing surface migrates faster than the leading surface. All the 


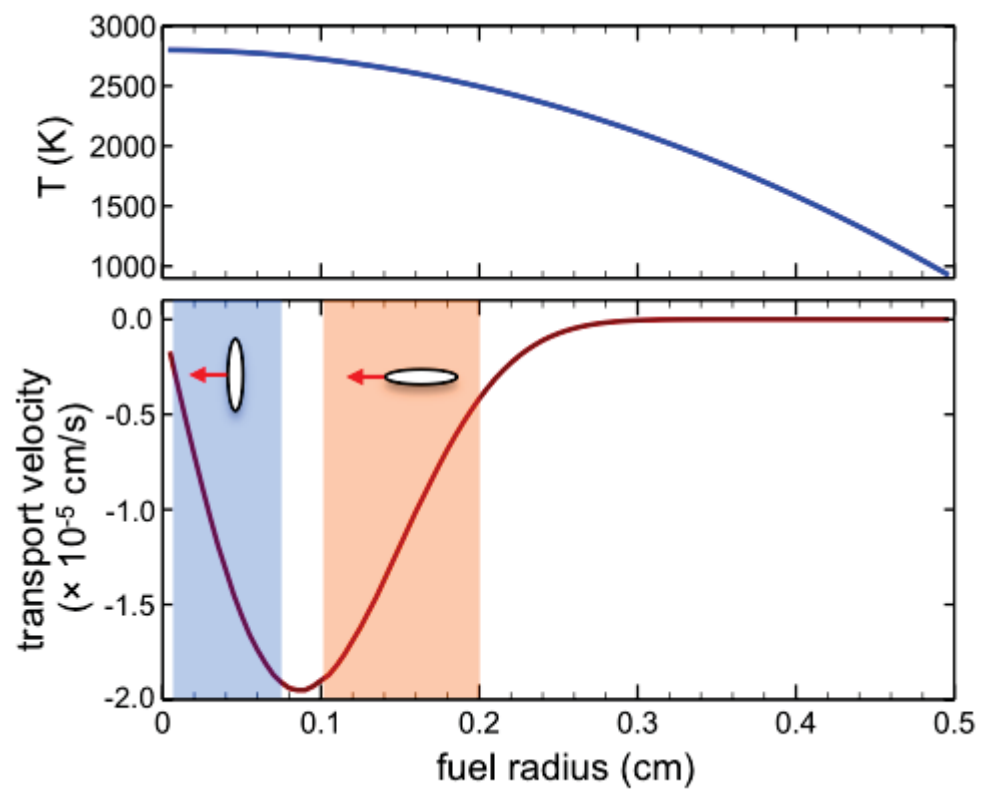

Figure 27: With an assumed temperature profile in the radial direction (top), the transport velocity of a pore due to the evaporation-condensation mechanism in the radial direction, as derived by Sens in REF.35, is plotted (bottom). Note that in these plots, the positive radial direction is along the $\mathrm{x}$-axis, and a negative transport velocity results in a pore migration toward the fuel centerline.

while, the concentration parameter $c$ remains strictly conserved, and the volume of the pore is also conserved. This kinetic driving force for pore shape change is countered by the increase in surface area of the pore, hence the pore's width in the x-direction does not reduce to zero. Rather, the pore assumes a steady-state lenticular morphology.

Each of the snapshots in Fig. 29 represent equally spaced intervals of time, with the top image corresponding to the beginning of the simulation. By inspection of the relative position of the pore at each instance of time, it can be deduced that the pore velocity is highest at the beginning of the simulation, while it is on the right side of the domain. In the later stages of the simulation, as the pore migrates to the left side of the domain, its velocity decreases. This is again due to the profile of the velocity field defined by Eq. 26, which is a negative-sloped line that equals zero at the left side of the domain. Hence, the overall pore velocity decreases as the pore approaches the left side of the domain.

We were interested to see if the change in the pore shape resulted in a deviation of the pore velocity from the prescribed transport velocity equation. To address this question, we calculated the instantaneous position of the pore's 'center of mass' by averaging the $\mathrm{x}$ - and y-positions of all the grid points with a value of $c>0.5$. These positions were plotted versus simulation time, which subsequently allowed us to calculate the pore's instantaneous velocity using a finite-difference approximation for the derivative of the pore position versus time curve. We then plotted the pore velocity versus the pore's x-position (i.e., the pore's radial position), with the results shown in Fig. 30. Aside from an initial acceleration that the pore must perform (the pore initially has a velocity of zero), the pore's velocity very closely matches the imposed transport velocity model, regardless of the fact that the pore has drastically changed its shape during migration. This result is in agreement with the work of Nichols [39], who concluded that the velocity of a migrating pore due to the vapor transport mechanism does not depend on the pore's shape. 

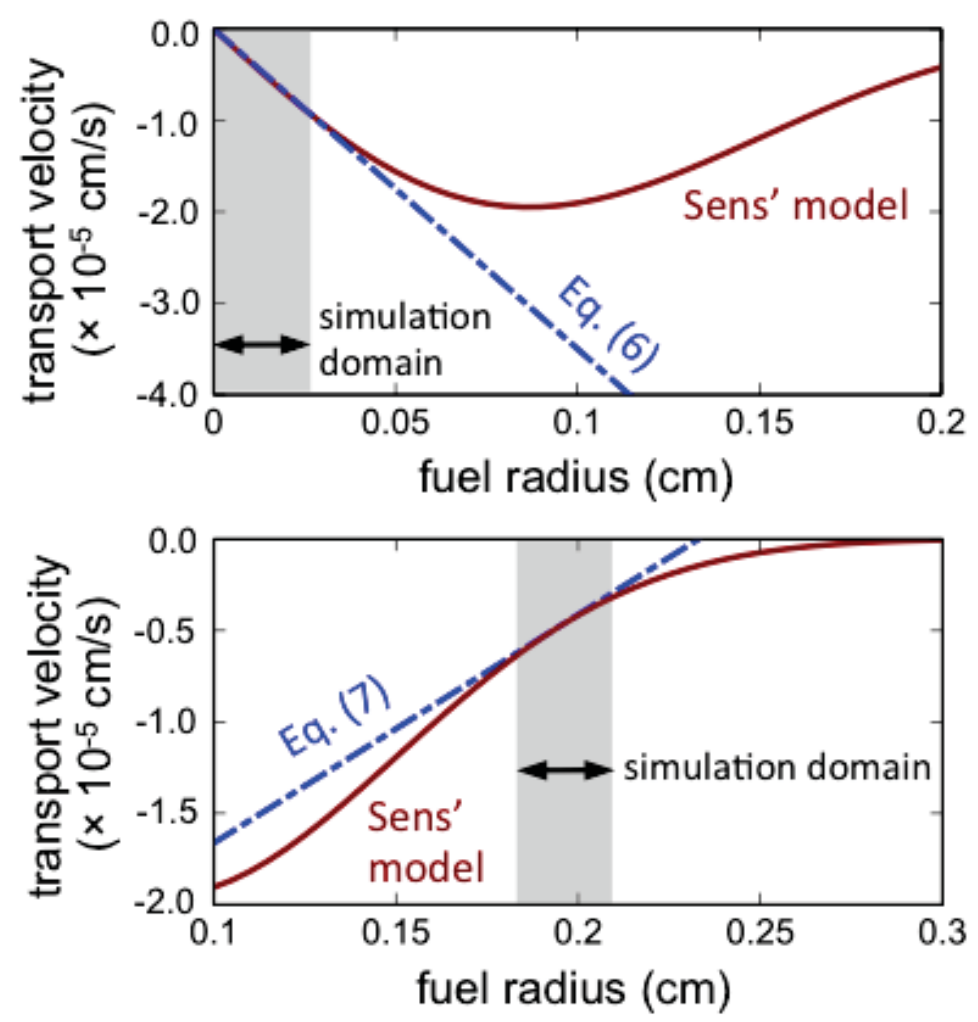

Figure 28: The simulations in this work used linear equations to describe the transport velocity in the radial direction. These linear equations overlap with the Sens' model in REF.35 at different radial locations, with the top plot showing Eq. 26 and the bottom plot showing Eq. 27. The grey shaded boxes correspond with the simulation domain size in the radial direction $(=256 \mu \mathrm{m}$, with a $512 \times 128$ grid).

Next, we prescribed the transport velocity expression given in Eq. 27, and Fig. 31 shows snapshots depicting the pore migration and morphology evolution. As opposed to the previous conditions, here the transport velocity consists of negative values throughout the domain (hence, as before, the pore migrates in the negative $\mathrm{x}$-direction), however the velocity field has a positive slope in the x-direction. This velocity field therefore induced a morphology change from a circular shape to a prolate (bullet) shape, which can be justified by the fact that the leading pore surface experiences a larger (i.e., a more negative) velocity than the trailing pore surface. This bulletshaped pore continues to evolve in shape as it migrates further towards the left-hand side of the domain. We observe that a somewhat circular 'head' develops at the leading surface of the pore, which seems to persist during migration. As before, we calculated the pore's velocity throughout the simulation, and plotted it versus the radial direction (Fig. 32), and again we observe that the change in pore shape does not result in a deviation in velocity from the prescribed model.

The dimensional changes observed in the migrating pores were calculated for both transport velocity profiles in terms of the pore's width in both the x-direction and y-direction. Figure33 shows these values plotted versus the pore's radial position. For both cases, the pores begin in the same position and with a radius of $15 \mu \mathrm{m}$. At the end of the simulation using Eq. 26, the lenticular pore reached $\mathrm{x}$ - and $\mathrm{y}$-dimensions of 10.5 and $31.5 \mu \mathrm{m}$, respectively. At the end of the simulation using Eq. 27, the bullet-shaped pore reached $\mathrm{x}$ - and $\mathrm{y}$-dimensions of 30.0 and $9.5 \mu \mathrm{m}$, 


\section{0}
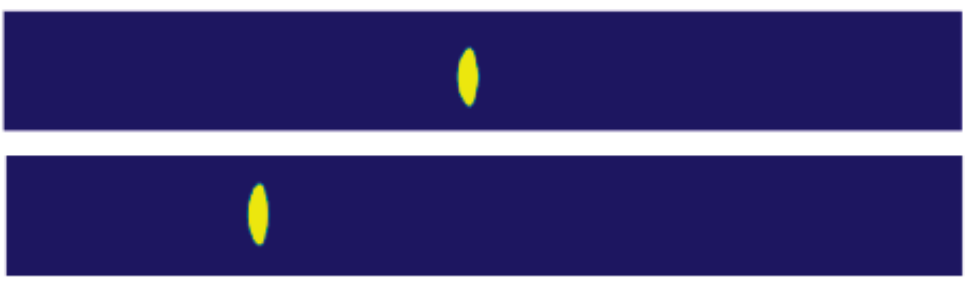

\section{1}

Figure 29: Simulation snapshots of pore migration using Eq. 26 as the transport velocity field. The top-to-bottom images correspond to 0, 1000, 2000, and 3000 simulation steps, respectively. The left side of the domain corresponds to the fuel centerline when using Eq. 26. These images were taken from a simulation that used the larger grid $(1024 \times 128)$.

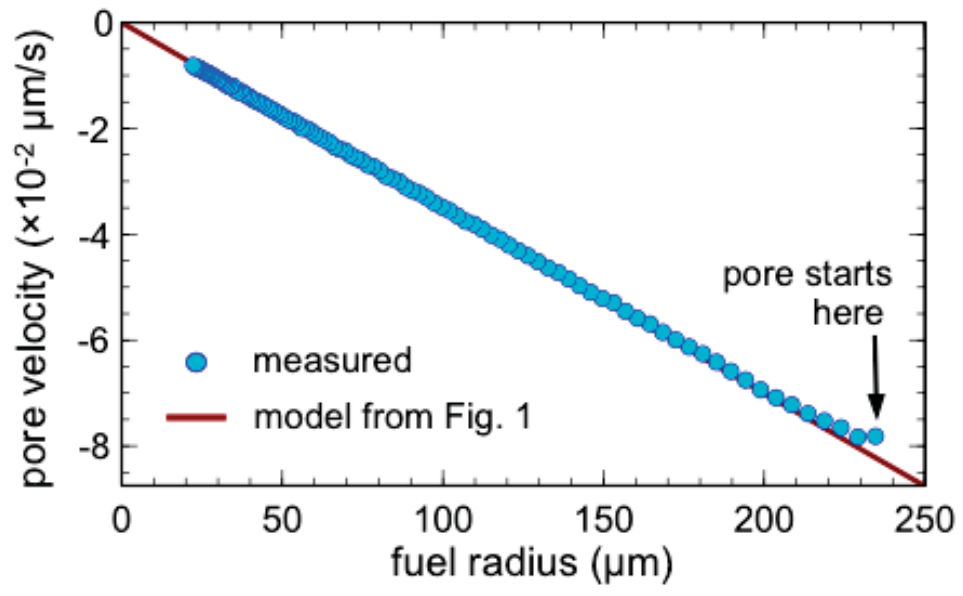

Figure 30: The velocity of a migrating pore is plotted versus the pore's radial position. The blue data points correspond to velocity values measured from a simulation, as explained in the tex, using Eq.26 for the transport velocity and a grid size of $512 \times 128$. Regardless of the fact that the pore has changed shape to a lenticular morphology, the pore velocity matches very closely to the prescribed model.

respectively. Once again, the only difference between the two simulations is the prescribed values for the transport velocity, and hence this important parameter (and its radial profile, including the sign of its slope) seems to dictate the change in pore shape during migration.

\subsection{Conclusions}

In summary, a phase-field model is presented to simulate pore migration due to the vapor transport mechanism of $\mathrm{UO}_{2}$ molecules through the pore interior due to a temperature gradient. The model utilizes an advection term that is appended to a conventional Cahn-Hilliard equation, within which 

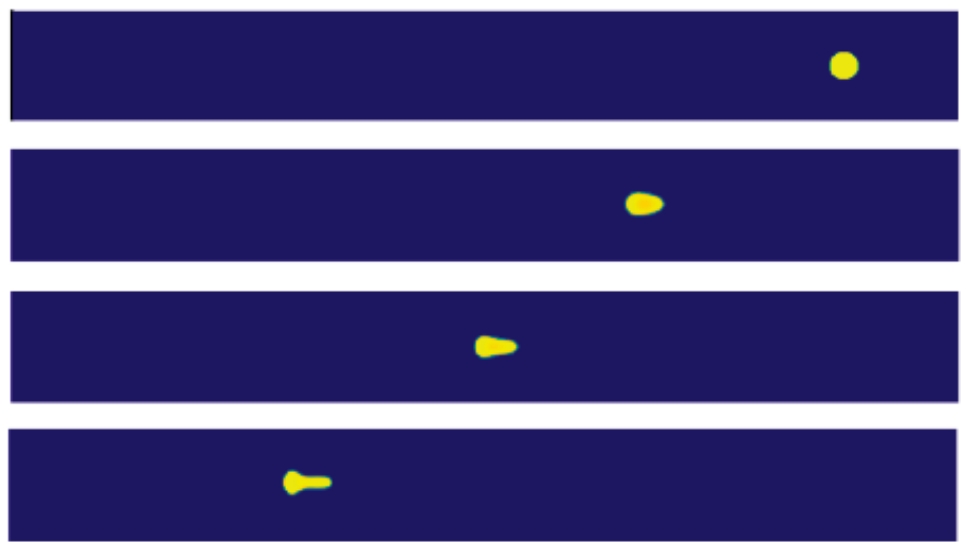

Figure 31: Simulation snapshots of pore migration using Eq. 27 as the transport velocity field. The top-to-bottom images correspond to 0, 1000, 2000, and 3000 simulation steps, respectively. These images were taken from a simulation that used the larger grid $(1024 \times 128)$.

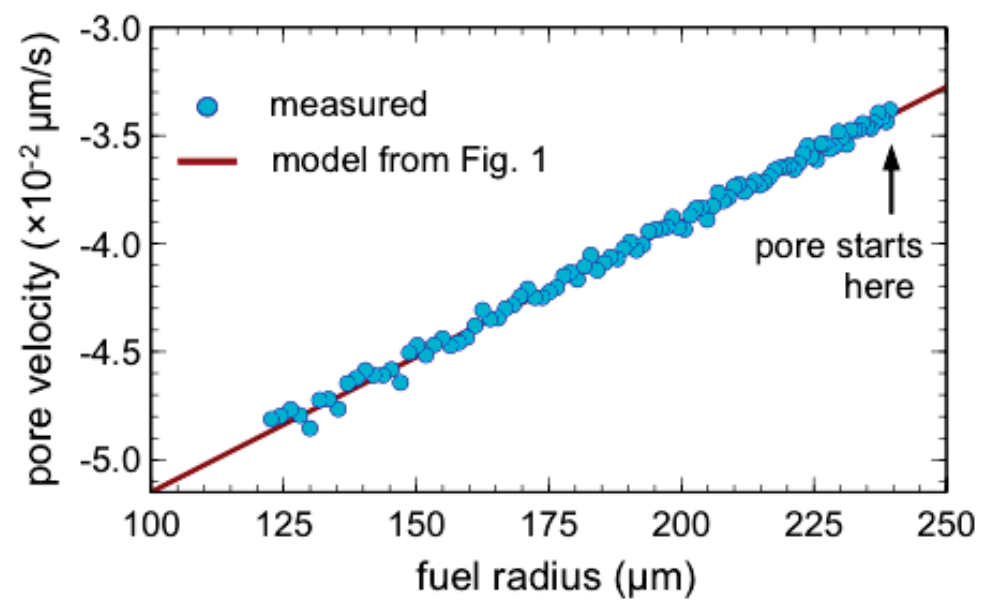

Figure 32: The velocity of a migrating pore versus the pore's radial position for a pore that transforms into a prolate (e.g., bullet) shaped morphology as shown in Fig. 30 when Eq. 27 is used as the transport velocity.

a vapor transport velocity field must be supplied. Here, we used two simple linear velocity profiles that closely correlate to the transport velocity equation developed by Sens [35] at two different radial positions within the fuel. Our simulations illustrate that the highly non-equilibrium shapes observed in migrating pores, namely lenticular and prolate geometries, can be explained by the slope of the transport velocity field in the radial direction. This gives additional support to the notion that large pore migration during fuel restructuring is driven by the vapor transport mechanism. In addition, we found that the magnitude of a migrating pore's velocity due to the vapor transport mechanism is independent of any changes that occur in the pore's shape. This conclusion agrees with that of Nichols [39], however, may be affected by the assumption built into Sens' model that the internal vapor pressure is solely a function of temperature - thus neglecting contributions from the surface tension.

Nevertheless, the successful reproduction of the transformation of an initially isotropic pore 

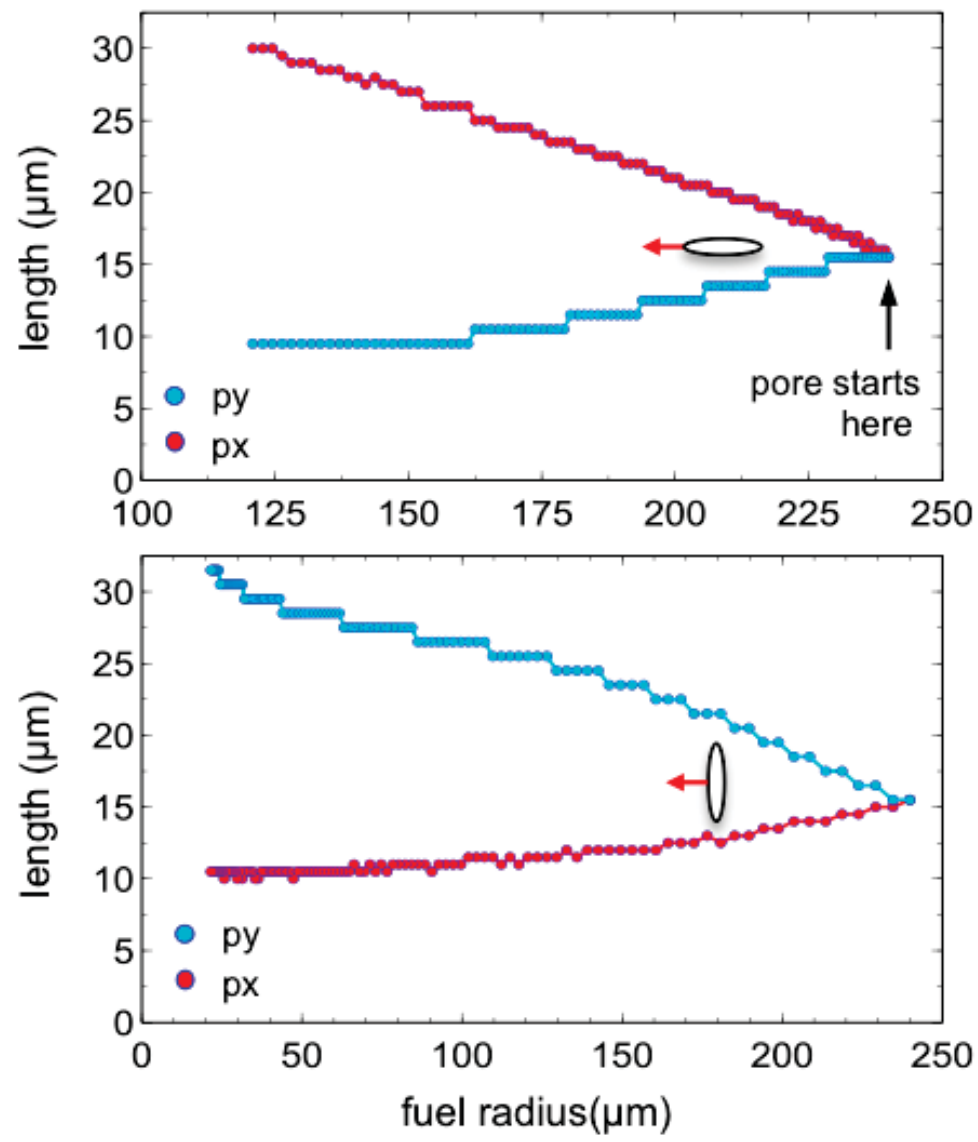

Figure 33: The $\mathrm{x}$ - and $\mathrm{y}$-dimensions of a migrating pore that transforms into (top) a prolate morphology and (bottom) a lenticular morphology. These values were measured from simulations using Eq. 27 and Eq. 26 for the top and bottom plots, respectively. The blue dots correspond to the pore's y-dimension and the red dots correspond to the pore's x-dimension.

into a lenticular shape or a prolate shape is an advance over recent attempts to simulate thermalgradient-driven pore migration [45, 46, 47]. Further refinement of this simulation model could include a direct calculation of the spatially-dependent temperature field surrounding the pore, and allow that to inform the transport velocity equation (here, we assumed a particular temperature profile when we assigned the transport velocity field). In addition, the migration of multiple pores could be simulated in a polycrystalline matrix to understand the formation of the columnar grain structure. We leave these efforts to future studies.

The current development is carried out using a in-house phase field code. The model will be implemented into MARMOT in FY2017. 


\section{Verification, validation and uncertainty quantification}

A more detailed discussion on verification, validation and uncertainty quantification has been given in previous assessment report with suggested data need $[4,5]$. Here some further opinion regarding the validation effort is elaborated. Compared with the burn-up based approach, the microstructure based fuel performance modeling approach allows for the inclusion of much richer physics and thus more rigorous validations. As the physics from various scales and areas are integrated together to predict the materials properties, a multiscale, multiphysics validation practice is required to fully assure the validity of these models.

The validation of the microstructure based models starts with the validations of fundamental material parameters/mechanisms, for example, fission gas atom diffusion coefficient/mechanism. Validations are also needed for the thermodynamic properties (structure, energy) and kinetic behaviors (mobility, transformation) of atomic scale microstructure features (bubbles, loops, interfaces). For this purpose, observations and measurements with atomic resolution, e.g., using high-resolution TEMs, are usually needed. Experiments that can extract these parameters/mechanisms are also useful.

The second level of validations focus on the sing-effect models, usually at the mesoscale. Examples include the effect gas diffusivity on homogeneous bubble formation and the effect of a certain type of point defects on thermal conductivity. The models are usually developed based on theories with assumptions, and may contain multiple material parameters/mechanisms. Well designed experiments are needed to exclude or quantify the effects of other factors.

Even after the single-effect models are validated, the combination of them into integrated materials models needs validations. For instance, the thermal conductivity model for UO2 contains the contributions from multiple microstructure features such as intrinsic defects, grain boundaries and so on. For each, single-effect validation is needed, followed by the validations of the overall model. Such validations usually need experiments at either the mesocale or macroscopic scale.

The engineering scale fuel performance modeling requires the usage of all relevant materials models, and also physics coupling between fuel behaviors and thermal hydraulic and neutronics. Therefore, validations are also desired when all these models are applied together. This last series of validations usually involve experiments at the fuel pin or the reactor level.

The models for microstructure evolution and structure-property relationship need different types of experiments. For the former, dynamic experiments are required to evolve the microstructure, with both the initial and final microstructure well characterized. In-situ observations are preferred although not always necessary. For the latter, microstructure characterization and property measurement can be done without evolving the material systems of interest. For both, the simulations and the experimental conditions should match each other as closely as possible during a validation practice. The data should be compared in details. A lot of times statistics is needed to capture the distribution of the data space. 


\section{Summary}

In FY16, significant progresses have been regarding research and development for oxide fuels at the lower-length-scale in three areas: 1) development of materials models to be used in engineering scale fuel performance modeling regarding the effect of lattice defects on thermal conductivity, 2) development of modeling capabilities for mesoscale fuel behaviors including stage- 3 gas release, grain growth, high burn-up structure, fracture and creep, and 3) improved understanding in material science by calculating the anisotropic grain boundary energies in $\mathrm{UO}_{2}$ and obtaining thermodynamic data for solid fission products. The results obtained have featured multiple journal publications and conference presentations including several invited talks. Much of the content in this report is expected to be published in peer-reviewed journals in a short future. Meanwhile, the progresses enhance the MARMOT capability for mesoscale fuel performance modeling of oxide fuels by advancing the models and extending the database of material parameters.

In the future, we will continue to perform multiscale modeling and simulation to develop microstructure-based fuel performance models. Many of the above areas take multiple years to be accomplished, with incremental success each year. In FY17, efforts will be made primarily to 1) improve the phase field algorithm in MARMOT towards quantitative prediction of oxide fuels, 2) further develop the grain growth capability in order to supply a mechanistic grain growth to BISON, 3) improve the grain scale fracture and creep model, 4) demonstrate high burn-up structure formation, 5) enhance the capability for hydrogen diffusion and hydride formation and 6) enable modeling of stage-3 fission gas release. Efforts will also be made on center-hole formation in fuels of fast reactors and fuel densification in light water reactors. As some MARMOT capabilities

are maturing, we will also collect experimental data and seek for experimental collaborations for validation. 


\section{References}

1. M.R. Tonks, D. Gaston, P.C. Millett, D. Andrs, and P. Talbot. An object-oriented finite element framework for multiphysics phase field simulations. Comp. Mat. Sci., 51(1):20-29, 2012.

2. D Gaston, C Permann, D Andrs, J Peterson, and A Slaughter. Moose enhancements towards delivery of an integrated framework. Technical report, Idaho National Laboratory, 2013.

3. R.L. Williamson, J.D. Hales, S.R. Novascone, M.R. Tonks, D.R. Gaston, C.J. Permann, D. Andrs, and R.C. Martineau. Multidimensional multiphysics simulation of nuclear fuel behavior. Journal of Nuclear Materials, 423:149 - 163, 2012.

4. M. R. Tonks, D. Schwen, Y. Zhang, P. Chakraborty, X. Bai, B. Fromm, J. Yu, and M. C. Teague. Assessment of marmot: A mesoscale fuel performance code. Technical report, Idaho National Laboratory, 2015.

5. D. Schwen, P. Chakraborty, L. K. Aagesen, Y. Zhang, K. Ahmed, D. A. Andersson, and C. Matthews. Assessment of marmot release 1.1: A mesoscale fuel performance code. Technical report, Idaho National Laboratory, 2016.

6. K. Ahmed, Y. Zhang, B. Biner, and M. Tonks. Uo2 grain growth: Developing phase field models for pore dragging, solute dragging and anisotropic grain boundary energies. Technical report, Idaho National Laboratory, 2016.

7. Y. Zhang, P. C. Millett, M. R. Tonks, X M Bai, and S B Biner. Molecular dynamics simulations of intergranular fracture in $\{\mathrm{UO} 2\}$ with nine empirical interatomic potentials. Journal of Nuclear Materials, 452(1-3):296 - 303, 2014.

8. X M Bai, Y Zhang, and MR Tonks. Testing thermal gradient driving force for grain boundary migration using molecular dynamics simulations. Acta materialia, 85:95-106, 2015.

9. Vasily V Bulatov, Bryan W Reed, and Mukul Jumar. Grain boundary energy function for fcc metals. Acta Mater., 37(7):1983 - 1993, 1989.

10. W T Read and W Shockley. Dislocation models of crystal grain boundaries. Physical Review, $78(3): 275-289,1950$.

11. Dieter Wolf. A read-shockley model for high-angle grain boundaries. Scripta Metall., 23:1713 $-1718,1989$.

12. S J Plimpton. Fast parallel algorithms for short-range molecular dynamics. J. Comp. Phys., 117:1-19, 1995.

13. CB Basak, AK Sengupta, and HS Kamath. Classical molecular dynamics simulation of uo2 to predict thermophysical properties. J. Alloys Compounds, 360:210-216, 2003.

14. P.V. Nerikar, K. Rudman, T.G. Desai, D. Byler, C. Unal, K.J. McClellan, simon R. Phillpot, Susan B. Sinnott, Pedro Peralta, Blas P. Uberuaga, and Christopher R. Stanek. Grain boundaries in uranium dioxide: Scanning electron microscopy ex- periments and atomistic simulations. Journal of the American Ceramic Society, 94:1893-1900, 2011.

15. N. Moelans, B. Blanpain, and P. Wollants. Quantitative analysis of grain boundary properties in a generalized phase field model for grain growth in anisotropic systems. Phys. Rev. B, 78:024113, 2008. 
16. K. Ahmed, Y. Zhang, B. Biner, and M. Tonks. High-burnup-structure (hbs): Model development in marmot for hbs formation and stability under radiation and high temperature. Technical report, Idaho National Laboratory, 2016.

17. A. Villani, E. P. Busso, and S. Forest. Field theory and diffusion creep predictions in polycrystalline aggregates. Modeling and Sim. in Mater. Sci. Eng., 23:055006, 2015.

18. JI Bramman, RM Sharpe, D Thom, and G Yates. Metallic fission-product inclusions in irradiated oxide fuels. Journal of Nuclear Materials, 25:201-215, 1968.

19. TB Massalski, H Okamoto, Subramanian PR, and L Kacprzak. Binary Alloy Phase Diagrams. American Society for Metals, Materials Park, Ohio, 1990.

20. HR Haines, PE Potter, and MH Rand. Some phase-diagram studies of systems with fissionproduct elements for fast reactor fuels. In International symposium on thermodynamics of nuclear materials. International Atomic Energy Agency, Vienna (Austria), 1980.

21. G Kresse and J Furthmuller. Efficient iterative schemes for ab initio total-energy calculations using a plane-wave basis set. Physical Review B, 54:11169-11186, 1996.

22. JP Perdew, K Burke, and M Ernzerhof. Generalized gradient approximation made simple. Physical Review Letters, 77:3865-3868, 1996.

23. JL Martins and A Zunger. Stability of ordered bulk and epitaxial semiconductor alloys. Physical Review Letters, 56:1400-1403, 1986.

24. M Zinkevich and N Mattern. Thermodynamic assessment of the mo-zr system. Journal of Phase Equilibria, 23:156-162, 2002.

25. RJ Perez and B Sundman. Thermodynamic assessment of the mo-zr binary phase diagram. CALPHAD, 27:253-262, 2003.

26. JB Darby Jr., DJ Lam, LJ Norton, and JW Downey. Intermediate phases in binary systems of technetium-99 with several transition elements. Journal of the Less Common Metals, 4:558-563, 1962.

27. M R Tonks, P C Millett, P Nerikar, S Du, D Andersson, C R Stanek, D Gaston, D Andrs, and $\mathrm{R}$ Williamson. Multiscale development of a fission gas thermal conductivity model: Coupling atomic, meso and continuum level simulations. Journal of Nuclear Materials, 440(1):193-200, 2013.

28. M. R. Tonks, D. Andersson, X. Y. Liu, D. Perez, A. Chernatynskiy, G. Pastore, C. R. Stanek, and R. Williamson. Development of a multiscale thermal conductivity model for fission gas in uo2. Journal of Nuclear Materials, 469:89-98, 2016.

29. J.K. Fink. Thermophysical properties of uranium dioxide. Journal of Nuclear Materials, 279:1$18,2000$.

30. X.M. Bai, M.R. Tonks, Y. F. Zhang, and J.D. Hales. Multiscale modeling of thermal conductivity of high burnup structures in uo2 fuels. Journal of Nuclear Materials, 470:208-215, 2016.

31. C. Hobson, R. Taylor, and J.B. Ainscough. Effect of porosity and stoichiometry on the thermal conductivity of uranium dioxide. J. Phys. D; Appl. Phys., 7:1003, 1974. 
32. S.G. Popov, J.J. Carbajo, V.K. Ivanov, and G. L. Yoder. Thermophysical properties of mox and uo2 fuels including the effect of irradiation. Technical report, Oak Ridge National Laboratory, 2000 .

33. DR Olander. Fundamental Aspects of Nuclear Reactor Fuel Elements. Technical Information Center, Energy Research and Development Administration, Oak Ridge, TN, 1976.

34. JR MacEwan and VB Lawson. Grain growth in sintered uranium dioxide: Ii, columnar grain growth. Journal of the American Ceramic Society, 45:42-46, 1962.

35. PF Sens. The kinetics of pore movement in uo2 fuel rods. Journal of Nuclear Materials, 43:293-307, 1972.

36. JK Platten. The soret effect: A review of recent experimental results. Journal of Applied Mechanics, 73:5-15, 2006.

37. FA Nichols. Theory of columnar grain growth and central void formation in oxide fuel rods. Journal of Nuclear Materials, 22:214-222, 1967.

38. FA Nichols. Pore migration in ceramic fuel elements. Journal of Nuclear Materials, 27:137-146, 1968.

39. FA Nichols. On the diffusional mobilities of particles, pores and loops. Acta Metallurgica, 20:207-214, 1972.

40. FA Nichols. On the thermal gradient migration of lenticular voids. Journal of Nuclear Materials, 84:319-326, 1979.

41. MV Speight. The migration of gas bubbles in material subject to a temperature gradient. Journal of Nuclear Materials, 13:207-209, 1964.

42. TG Desai, PC Millett, M Tonks, and D Wolf. Atomistic simulations of void migration under thermal gradient in uo2. Acta Materialia, 58:330-339, 2010.

43. H Kawamata, H Kaneko, H Furuya, and M Koizumi. Migration rate of lenticular voids in uo2 under the influence of temperature gradient. Journal of Nuclear Materials, 68:48-53, 1977.

44. SY Hu and $\mathrm{CH}$ Henager. Phase-field simulation of void migration in a temperature gradient. Acta Materialia, 58:3230-3237, 2010.

45. Y Li, SY Hu, X Sun, F Gao, CH Henager, and M Khaleel. Phase-field modeling of void migration and growth kinetics in materials under irradiation and temperature field. Journal of Nuclear Materials, 407:119-125, 2010.

46. L Zhang, MR Tonks, PC Millett, Y Zhang, K Chockalingam, and SB Biner. Phase-field modeling of temperature gradient driven pore migration coupling with thermal conduction. Computational Materials Science, 56:161-165, 2012.

47. V Tikare and EA Holm. Simulation of grain growth and pore migration in a thermal gradient. Journal of the American Ceramic Society, 81:480-484, 2005.

48. LQ Chen. Phase-field models for microstructure evolution. Annual Review of Materials Research, 32:113-140, 2002. 
49. S Rokkam, A El-Azab, PC Millett, and D Wolf. Phase field modeling of void nucleation and growth in irradiated metals. Modelling and Simulation in Materials Science and Engineering, 17:064002, 2009.

50. P C Millett, A El-Azab, S Rokkam, M Tonks, and D Wolf. Phase-field simulation of irradiated metals: Part i: Void kinetics. Computational Materials Science, 50(3):949-959, 2011.

51. LQ Chen and J Shen. Applications of semi-implicit fourier-spectral method to phase field equations. Computer Physics Communications, 108:147-158, 1998. 